Version postprint. Published in Cahiers de Nutrition et de Diététique :

https://doi.org/10.1016/j.cnd.2021.04.002

\title{
Participation à un jardin collectif urbain et santé : revue systématique de la littérature
}

Titre en anglais: Urban collective garden participation and health: a systematic literature review

Auteurs : Marion Tharrey, Nicole Darmon

MOISA, Univ Montpellier, CIRAD, CIHEAM-IAMM, INRAE, Institut Agro, Montpellier, France

Responsable de la correspondance : Marion Tharrey

Adresse : INRAE Montpellier, UMR MOISA Bât. 26, 2 place Pierre-Viala, 34060

Montpellier Cedex 1, France

Mail: mariontharrey@hotmail.fr,

Téléphone : +33 622397272.

\section{RÉSUMÉ EN FRANÇAIS}

De nombreuses villes implantent des jardins collectifs sur leurs territoires dans le but de promouvoir la santé et le bien-être des habitants. Nous avons conduit une revue systématique de la littérature des études s'appuyant sur des méthodes quantitatives pour explorer le lien entre participation à jardin collectif et santé des citadins adultes. Sur les 1430 articles identifiés, 18 ont été retenus. L'outil «d'évaluation de la qualité des études quantitatives » développé par 1'EPHPP a été utilisé pour évaluer la qualité méthodologique des études. La participation à un jardin collectif était associée à une plus grande consommation de fruits et légumes, ainsi qu’à un bien-être mental et social accru. Les résultats étaient mitigés pour l'IMC, l'activité physique 
et d'autres indicateurs de la santé physique. Les études étaient de faible qualité, principalement en raison de leur design transversal $(n=14 / 18)$ et de leur risque élevé de biais de sélection ( $\mathrm{n}$ $=15 / 18)$. Davantage d'études longitudinales avec évaluation avant/après et présence d'un groupe contrôle sont nécessaires pour déterminer si les jardins collectifs peuvent être un outil efficace pour promouvoir la santé des citadins.

Mots clés (3 à 5) : fruits et légumes; activité physique ; bien-être ; jardins partagés ; jardins familiaux.

\section{RÉSUMÉ EN ANGLAIS}

Collective gardens are developing in many cities as a tool to promote the health and well-being of urban dwellers. We conducted a systematic review of the literature of studies using quantitative methods to explore the relationship between participation in community gardens and health of urban adults. Of 1,430 articles identified, 18 were included in the systematic review. Methodological quality was assessed using the Quantitative Study Quality Assessment Tool developed by the EPHPP. Collective gardening was associated with higher fruit and vegetable consumption, as well as better mental and social health. Mixed results were found for BMI, physical activity and other physical health outcomes. The methodological quality of the studies was weak, mainly due to their cross-sectional design $(n=14 / 18)$ and high risk of selection bias $(\mathrm{n}=15 / 18)$. More longitudinal studies with pre-post evaluation and presence of a control group are needed to determine whether community gardens can be an effective tool to promote the health of city dwellers.

Key words (3 to 5 key words or phrases); fruit and vegetables; physical activity; well-being; community garden; allotment garden. 
52

53

- Une revue systématique de la littérature sur le lien entre jardin collectif et santé a été réalisée

- Le jardin collectif est associé à la consommation de fruits et légumes, ainsi qu'à la santé mentale et sociale

- Les études existantes sont majoritairement transversales

- La seule étude suivant les jardiniers sur le long terme (1 an) n'a pas trouvé d'effet du jardinage collectif sur la santé

- Davantage d'études longitudinales, de type expérimental ou quasi-expérimental, sont nécessaires 
61

62

\section{INTRODUCTION}

Les espaces verts urbains sont aujourd'hui reconnus comme un élément clé du développement durable des villes européennes en raison de leurs multiples impacts potentiels sur la santé, les relations sociales et l'environnement $(1,2)$. Parmi les différentes formes d'espaces verts, l'intérêt croissant des citadins quant à la possibilité de cultiver ses propres fruits et légumes (3) a favorisé l'essor des jardins potagers en ville. De nombreux bénéfices liés à la pratique même du jardinage (tous formats de jardins confondus) ont été décrits notamment sur la santé mentale : diminution des troubles de l'humeur, de l'anxiété et de la dépression, amélioration de la qualité de vie, des fonctions cognitives ou encore de la satisfaction vis-à-vis de sa propre existence $(4,5)$. Néanmoins, les preuves de l'impact positif du jardinage sur la santé proviennent principalement d'interventions menées dans des milieux institutionnalisés (écoles, hôpitaux, centres de soins, maisons de retraite etc.) (6-10) et peu d'études ont été conduites en population générale urbaine. Récemment, une méta-analyse a conclu que le jardinage pratiqué par des adultes vivant en population générale avait un effet bénéfique sur l'IMC, la nutrition et la santé physique par rapport à des non-jardiniers (11). En particulier, la littérature suggère que les jardins collectifs (espaces cultivés à distance du domicile et gérés collectivement par un groupe de jardiniers (12)), en agissant sur de multiples déterminants, auraient le potentiel d'améliorer différentes dimensions de la santé, et notamment, du fait de leur caractère collectif, la santé sociale (13). Les formes les plus courantes de jardins collectifs sont les jardins partagés et les jardins familiaux. Les jardins partagés sont des espaces cultivés collectivement et ayant pour principale vocation de favoriser les liens sociaux et les échanges intergénérationnels entre les habitants d'un quartier, ainsi que de sensibiliser à la biodiversité $(15,16)$. Les parcelles peuvent être cultivées collectivement ou individuellement et sont généralement de petite taille $\left(\leq 20 \mathrm{~m}^{2}\right.$ pour les parcelles individuelles) (17). Les jardins familiaux sont des terrains subdivisés en grandes parcelles (de 100 à $500 \mathrm{~m}^{2}$ ) louées à une personne ou à une famille afin qu'elle puisse 
y cultiver ses propres fruits et légumes et autres produits potagers, mais aussi à des fins récréatives $(18,19)$. Plus grands que les jardins partagés, les jardins familiaux sont généralement situés en périphérie des villes. Les revues de la littérature existante soulignent la multifonctionnalité des jardins collectifs, qui permet l'accès à des fruits et légumes frais tout en offrant des espaces de loisirs, de détente et de cohésion sociale. Néanmoins, ces revues de littérature ne font pas de distinction entre les résultats des études quantitatives et qualitatives $(13,20-24)$, et/ou ciblent un pays spécifique $(20,25,26)$, une population spécifique (24) ou un seul paramètre de santé $(22,25)$, et/ou n'ont pas été conduites sous la forme d'une recherche systématique de la littérature $(14,27)$, ou encore traitent des jardins urbains de manière plus générale $(22,23,28)$. En outre, aucune étude n'a fourni d'indication sur l'intensité et la durée du temps passé à jardiner.

Nous avons donc conduit une revue systématique de la littérature afin d'identifier et d'évaluer de manière critique toutes les études quantitatives ayant analysé la relation entre la participation à un jardin collectif et l'état de santé des jardiniers en ville. Nous avons considéré la santé comme un état de complet bien-être physique, mental et social, tel que défini par l'OMS (29). 


\section{MATERIEL ET METHODES}

Cette revue systématique de la littérature a été effectuée conformément aux lignes directrices PRISMA (Preferred Reporting Items for Systematic Reviews and Meta-analyses) (30).

\section{Stratégie de recherche}

Une recherche bibliographique systématique a été effectuée à l'aide des bases de données PubMed et Web Of Science selon la combinaison de termes suivante : (“collective garden" OR "community garden" OR “urban garden" OR "shared garden” OR “allotment garden”) AND (“health” OR “well-being” OR “nutrition” OR “diet” OR “physical activity” OR “social” OR “leisure” OR “vegetable” OR “fruit” OR “stress” OR “depression” OR “anxiety”).

\section{Critères d'inclusion et d'exclusion}

Les critères PICOS (Population, Intervention, Comparison, Outcomes, Study) ont été utilisés pour guider l'identification des études éligibles pour la présente revue de la littérature (Tableau 1). Tous les articles scientifiques originaux anglophones publiés dans des revues à comité de lecture entre janvier 2000 et décembre 2020, utilisant des méthodes quantitatives, et faisant état d'un ou plusieurs résultats de santé associés à la participation à un jardin partagé en population générale adulte urbaine ont été inclus. Nous avons exclu de l'analyse les études $i$ ) faisant référence au jardinage en général ou à d'autres formes de jardinage non entrepris dans un jardin collectif (jardins privés, ornementaux, etc.) ; ii) évaluant des programmes de jardinage réalisés en milieux institutionnalisés (écoles, hôpitaux, maisons de retraite, centres de santé, prisons, camps de réfugiés) ; iii) ciblant les individus âgés de moins de 18 ans ; iv) réalisées 
dans des zones rurales ou dans des pays du sud; ou v) basées uniquement sur des approches qualitatives. Les analyses documentaires, les thèses et les mémoires, les lettres à l'éditeur, les chapitres de livres et les commentaires d'auteurs ont été exclus de l'analyse. Sur la base de la définition du Bureau du recensement des États-Unis (2000), nous avons considéré les jardins comme étant urbains s'ils étaient situés dans des zones ayant une densité de population d'au moins 2500 habitants $/ \mathrm{km}^{2}$ et une population totale minimale de 50000 habitants (31).

\section{Stratégie de recherche et extraction des articles scientifiques}

La Figure 1 présente le diagramme PRISMA décrivant le processus d'identification, de criblage et de sélection des articles. La recherche bibliographique a été menée par MT et a conduit à l'identification de 1430 articles (après élimination des doublons). La sélection a ensuite été effectuée en trois phases. Tout d'abord, le titre et le résumé des études ont été parcourus pour vérifier qu'ils répondaient bien aux critères d'inclusion et d'exclusion. Cette étape a conduit à l'exclusion de 1385 articles. En cas de doute, l'article a été conservé pour un examen plus approfondi. Dans un second temps, les articles restants ont été lus dans leur intégralité et 30 d'entre eux ont été exclus, conduisant à 15 articles intégraux. Notons que parmi ces 15 articles, l'un portait sur une étude réalisée «en milieu urbain » sans précision supplémentaire (32), un autre portait sur des jardiniers habitant majoritairement - mais pas exclusivement - en zone urbaine (33), et un troisième portait sur un échantillon comprenant des personnes participant à un jardin partagé et/ou à des projets d'embellissement de leur quartier, la majorité des participants étant impliqués dans les deux activités (34). De plus, à partir des références des articles sélectionnés, et sur la base de notre expertise, trois articles supplémentaires $(32,35,36)$ ont été ajoutés conduisant à un total de 18 articles. 
Pour chaque article, les informations suivantes ont été extraites : auteurs, année, lieu,

150

151

152

153

154

155

156

157

158

159

160

161

162

163 caractéristiques des participants, taille de l'échantillon, design d'étude, données collectées, outils de mesure, résultats en matière de santé, ancienneté dans le jardin et fréquence de jardinage (Tableau 2).

\section{Évaluation de la qualité méthodologique des études}

La qualité des études incluses a été évaluée à l'aide de l'outil d'évaluation de la qualité des études quantitatives développé par l' EPHPP (Effective Public Health Practice Project) (37). L'outil EPHPP évalue les six domaines suivants : biais de sélection, design de l'étude, facteurs de confusion, conduite ou non en aveugle, méthode de collecte des données et taux d'attrition. Conformément aux directives de l'outil, chaque domaine a été jugé comme étant de «bonne », « moyenne » ou « faible » qualité. L'évaluation de la qualité a été réalisée indépendamment par les deux auteurs (MT et ND) et, en cas de désaccord, une décision a été prise par consensus.

\section{RÉSULTATS}

\section{Caractéristiques des études}

Concernant les zones géographiques, sur les 18 études incluses dans l'analyse, huit ont été conduites aux États-Unis (32,34,35,38-42), huit en Europe (36,43-47), une au Japon (48) et une à Singapour (49). Deux ont été conduites en France : l'une dans des quartiers défavorisés de Marseille (46), et l'autre dans les jardins partagés de la ville de Montpellier (45). Les études menées aux États-Unis, en France et à Singapour ciblaient des jardins partagés $(\mathrm{n}=11)$ $(32,34,49,35,38-42,45,46)$, et les autres, des jardins familiaux $(n=7)(33,36,43,44,47,48,50)$. 
171 Deux études ont été menées auprès de personnes âgées $(33,43)$ et trois auprès de populations défavorisées $(32,39,46)$. La moyenne d'âge des jardiniers allait de 41 à 66 ans et le nombre moyen de jardiniers jardinant dans un jardin collectif était de 87 (écart : 21 - 271).

Concernant le design des études, 14 sur les 18 avaient un design transversal (i.e. études descriptives permettant d'observer un événement à un instant donné) (32-36,38-41,43,46-49). Les quatre autres études étaient basées respectivement sur : une évaluation post-test uniquement avec présence d'un groupe de comparaison (i.e. études dans lesquelles les données ne sont recueillies qu'après intervention) (42), une évaluation avant-après une session de jardinage avec présence d'un groupe de comparaison (44), un essai contrôlé randomisé évaluant l'effet d'une session de jardinage (50), et, pour l'étude conduite à Montpellier, une expérimentation naturelle évaluée selon un design quasi-expérimental (c'est à dire une évaluation avant-après avec groupe de comparaison mais sans randomisation) (45). Un essai contrôlé randomisé suivant les jardiniers pendant 1 an a été identifié, a été identifié (51) mais les résultats n'étant pas encore publiés, cette étude n'a pas été incluse dans la présente revue de la littérature.

Concernant l'échantillonnage, deux études ont utilisé les données d'une enquête représentative de la population générale $(34,38)$, quatre ont utilisé un échantillonnage aléatoire de type multi-stage pour augmenter la proportion des jardiniers $(35,40,41,47)$, et, dans l'étude quasi-expérimentale conduite à Montpellier, tous les nouveaux jardiniers débutant dans un jardin partagé en 2018 ont été contactés (45). Les autres études étaient basées sur des échantillons de convenance.

Concernant les groupes de comparaison, ils étaient composés soit de non-jardiniers $(32,33,38,42,44-46,48)$, soit de jardiniers possédant un jardin privé $(39,47)$, ou des deux $(35,40,41)$. Deux études comparaient des jardiniers jardinant dans un jardin collectif ou privé à des non-jardiniers engagés dans des activités physiques autres que le jardinage $(43,49)$. Une autre étude a comparé la participation des membres de foyers à un jardin partagé ou à une activé 
d'embellissement du quartier à la participation à des réunions de quartier ou à l'absence de participation à ces différentes activités (34). Une étude n'avait pas de groupe contrôle (36). Enfin l'unique essai contrôlé randomisé a comparé le bénéfice santé de 30 min de jardinage par rapport à 30 min de lecture dans l'abri de jardin (50).

Concernant les variables de santé, au sein des 18 articles retenus, la santé physique était la principale dimension santé étudiée $(n=11)$ au travers de l'alimentation $(n=6)$, de l'anthropométrie $(n=6)$, de l'activité physique $(n=4)$ ou d'autres variables de santé physique $(n=3)$. Les autres aspects de la santé étudiés étaient la santé mentale $(n=10)$ et la santé sociale $(n=7)$. Enfin, quatre études sur les 18 ont utilisé des méthodes de mesure objectives $(43,45,46,50)$

\section{Jardins collectifs et alimentation}

Six études ont examiné l'alimentation des jardiniers $(32,38,39,41,45,46)$, dont trois menées au sein de populations défavorisées $(32,39,46)$. Toutes les études étaient transversales $(32,38,39,41,46)$ excepté l'étude quasi-expérimentale conduite à Montpellier (45). Les variables étudiées étaient la consommation de fruits et légumes $(32,41)$ ou de légumes uniquement (39), les approvisionnements alimentaires mensuels des foyers $(45,46)$, la consommation de sodas (32), ou encore la fréquence d'utilisation de la restauration rapide (32). Les études ont généralement utilisé comme outils de collecte de données des questionnaires standardisés (tels que le BRFSS $(32,41)$ ou le questionnaire alimentaire de l'EFNEP (39)) ou des questionnaires de fréquence de consommation (32). Les deux études réalisées en France ont opté pour une mesure objective des approvisionnements alimentaires mensuels des foyers (collecte des tickets de caisse alimentaires couplée à la tenue de registres alimentaires sur une période d'un mois) $(45,46)$. 
Toutes les études transversales ont trouvé une relation positive entre la participation à un jardin collectif et la consommation de fruits et légumes $(32,38,39,41,46)$. Parmi elles, une étude n'a pas observé de différences significatives de fréquences de consommation de fruits, de sodas et d'utilisation de la restauration rapide entre jardiniers et non-jardiniers (32). Dans une étude menée au sein d'une population d'adultes défavorisés fréquentant un jardin (privé ou partagé) aux États-Unis, les jardiniers ont déclaré avoir doublé leur consommation de légumes grâce au jardin, atteignant ainsi les 2,5 portions quotidiennes recommandées (39). Dans une autre étude, les jardiniers jardinant dans un jardin collectif ont déclaré consommer des fruits et légumes environ une fois de plus par jour que des non-jardiniers ou que des personnes jardinant dans un jardin privé (41). Aux États-Unis, dans une étude transversale basée sur une enquête représentative en population générale, des individus appartenant à un foyer dont au moins un membre participait à un jardin collectif avaient, par rapport à des individus issus de ménages de non-jardiniers, une consommation quotidienne de fruits et légumes multipliée par 1,4 en moyenne et étaient 3,5 fois plus susceptibles de consommer des fruits et légumes au moins 5 fois par jour (38). Dans l'étude transversale réalisée à Marseille, les approvisionnements alimentaires des foyers de jardiniers contenaient plus de fruits et de légumes que ceux des nonjardiniers (+158 g/j.pers), alors qu'aucune différence n'était observée pour les autres groupes alimentaires (46). Cette différence de consommation de fruits et de légumes était principalement due aux quantités achetées, et non aux quantités produites dans le jardin qui atteignaient seulement $28 \mathrm{~g} / \mathrm{j}$ et par personne du foyer en moyenne, en incluant les pommes de terre et les légumineuses (46). L'étude quasi-expérimentale réalisée à Montpellier a confirmé les faibles quantités produites dans les jardins partagés (en moyenne $14 \mathrm{~g} / \mathrm{j}$ de fruits et légumes et par personne du foyer) mais n'a pas observé d'impact de la première année sur les approvisionnements en fruits et légumes ni sur les parts de dépenses dans les différents groupes alimentaires (45). 
Jardins collectifs et anthropométrie

Six études ont évalué 1'IMC des jardiniers $(35,39,42,43,45,48)$. Quatre d'entre elles

étaient transversales $(35,39,43,48)$. L'IMC a été estimé à partir du poids et de la taille déclarés par les participants dans cinq études $(35,39,42,45,48)$, tandis qu'une étude a utilisé des mesures objectives (43).

Une seule étude transversale sur les quatre a observé un IMC plus faible chez les jardiniers que chez les non-jardiniers (35). Les autres études transversales n'ont pas trouvé de différence significative d'IMC entre les jardiniers et le groupe de comparaison, que celui-ci soit constitué de jardiniers jardinant dans un jardin privé (39), de non-jardiniers (48), ou d'individus participants à d'autres formes d'activité physique (43). Dans les études non-transversales, l'étude basée sur une évaluation post-test uniquement, a montré que les jardiniers jardinant dans un jardin collectif avaient un IMC inférieur à celui de leurs frères et sœurs ou voisins du même sexe (allant de $-2,36$ à $-1,33$ unités d'IMC), tandis qu'aucune différence significative n'était observée avec les conjoints des jardiniers (42). Enfin, dans l'étude quasi-expérimentale réalisée à Montpellier, l'IMC des jardiniers était plus faible que celui des non-jardiniers à l'entrée dans le jardin, et a significativement augmenté (et de façon comparable) dans les deux groupes au bout d'un an, mais cette augmentation n'était pas liée à la première année de jardinage qui n'a eu aucun impact spécifique sur l'IMC des jardiniers (45). Notons que des mesures anthropométriques autres que l'IMC n'ont pas été explorées dans les études existantes.

\section{Jardins collectifs et activité physique}


de l'IPAQ ((International Physical Activity Questionnaire) (43), ou deux items du SQUASH (Short QUestionnaire to ASsess Health enhancing physical activity) (33), ou encore deux items évaluant la fréquence d'activité physique modérée et vigoureuse (32). Dans l'étude quasiexpérimentale réalisée à Montpellier, l'activité physique a été mesurée objectivement à l'aide d'accéléromètres (45).

Dans les études transversales, deux études sur les trois, l'une menée au sein d'une population défavorisée (32) et l'autre au sein d'une population âgée (43), n'ont pas trouvé de différence d'activité physique entre jardiniers et non jardiniers. A l'inverse une étude a montré que les jardiniers (qu'ils soient âgés de plus ou moins 62 ans) déclaraient pratiquer une activité physique modérée plus fréquemment que leurs voisins, bien que cette différence ne soit observée que pendant l'été (33). Enfin, l'étude quasi-expérimentale réalisée à Montpellier n'a pas observé d'effet de la première année de participation à un jardin collectif sur la dépense énergétique liée à l'activité physique, ni sur le temps passé dans des activités de différentes intensités (45).

\section{Jardins collectifs et autres variables de santé physique}

Quatre études, toutes transversales, ont analysé d'autres indicateurs de la santé physique tel que l'état de santé général perçu $(4,32,33,43)$, les problèmes de santé déclarés $(4,33)$, les contraintes physiques (c'est-à-dire lorsque l'état de santé limite la réalisation de plusieurs activités quotidiennes d'intensité faible à vigoureuse) (33), la fréquence des consultations chez le médecin (33), la pression artérielle (43), ou encore la fonction pulmonaire (43). La santé générale perçue a été mesurée à l'aide de questionnaires standardisés basés sur un seul item $(4,32,33)$ ou sur la sous-partie du SF-36v2 Health Survey portant sur la santé physique (43). 
chez le médecin ont été déclarés par les participants $(4,33)$. La pression artérielle et la fonction pulmonaire ont été mesurées (43).

Deux études ont trouvé une association positive entre la participation à un jardin collectif et la santé physique $(33,48)$. En ce qui concerne la santé générale perçue, une étude a montré que les jardiniers déclaraient avoir une meilleure santé générale et moins de problèmes de santé que les non-jardiniers (48), tandis qu'une autre étude n'a pas trouvé de différence significative concernant la santé générale perçue (32). Dans une étude distinguant les jardiniers de 62 ans ou plus, ces derniers obtenaient de meilleurs résultats que leurs voisins non-jardiniers de la même tranche d'âge en ce qui concerne les contraintes physiques (limitation de l'activité locomotrice), les problèmes de santé déclarés et la fréquence des consultations chez le médecin, tandis qu'aucune différence n'a été constatée entre les jardiniers de moins de 62 ans et leurs voisins du même âge (33). Enfin, aucune différence de pression artérielle, de fonction pulmonaire ou de santé physique générale n'a été observée entre des personnes âgées jardinant dans un jardin collectif et celles ayant un jardin privé ou participant à d'autres formes d'activité physique (43).

\section{Jardins collectifs et bien-être mental}

Dix études, dont sept transversales, ont examiné la santé mentale des jardiniers à travers divers indicateurs tels que le stress $(33,43,47,49,50)$, la détresse psychologique $(32,48)$, la satisfaction vis-vis de sa propre existence $(32,33)$, le bonheur subjectif (36), l'humeur (50), le bien-être psychologique $(32,45,49)$, l'autonomisation (32), la santé mentale générale (43), l'estime de soi (49), la résilience (49), l'optimisme (49), l'ouverture (49), les effets réparateurs du jardin (47), ou encore la perception et l'acceptation de son corps (44). Les études se sont appuyées sur des mesures subjectives à partir de questionnaires, à l'exception de l'essai contrôlé 
randomisé qui était basé sur une mesure du cortisol salivaire, un biomarqueur endocrinien robuste du stress (50).

Toutes les études transversales ont fait état d'au moins une association positive entre la participation à un jardin collectif et un indicateur de la santé mentale $(32,33,36,43,47-49)$. Une étude conduite au sein d'une population défavorisée a montré que les jardiniers avaient un plus grand bien-être psychologique et une plus faible détresse psychologique que les non-jardiniers, bien qu'aucune différence significative n'ait été observée entre les groupes en ce qui concerne l'autonomisation et la satisfaction vis-à-vis de sa propre existence (32). De même, une étude a montré que les jardiniers avaient des niveaux de bien-être subjectif, de résilience et d'optimisme plus élevés que chez les non-jardiniers, tandis qu'il n'y avait pas, entre les groupes, de différence de stress, d'estime de soi ou d'ouverture (49). Dans une autre étude, la détresse psychologique évaluée à l'aide du Questionnaire de Santé Générale (une mesure commune des troubles psychiatriques mineurs dans la population générale) était plus faible chez les jardiniers que chez les non-jardiniers (48). Une étude comparant différents niveaux de fréquentation des jardins collectifs a observé qu'une plus grande fréquentation était associée à un bonheur subjectif accru chez les jardiniers (36). Le jardinage a également été associé à des effets dits « réparateurs » tels que la réduction du stress liés à la présence de milieux naturels (47). En comparant des jardiniers de différents groupes d'âge, une étude a montré que les jardiniers de 62 ans et plus déclaraient moins de stress et une meilleure satisfaction vis-à-vis de leur propre existence que les jardiniers plus jeunes et que leurs voisins non-jardiniers (33). La pratique du jardinage dans un jardin collectif a également été associée, par rapport à de l'exercice physique en salle, à un stress perçu moindre chez les personnes âgées, bien qu'il n'y avait pas de différence de santé mentale générale entre les groupes (43). Notons aussi que le jardinage peut parfois être une source de stress, néanmoins celui-ci est plus faible chez les jardiniers jardinant dans des jardins collectifs que chez ceux jardinant dans leur jardin privé (47). 
Deux études ont évalué les impacts à court terme d'une session de jardinage, au travers

d'un essai contrôlé randomisé (50) et d'une étude avant-après avec groupe de comparaison (44).

Dans l'essai contrôlé randomisé, les jardiniers, après avoir effectué une tâche stressante, pratiquaient soit une séance de 30 minutes de jardinage en plein air, soit une séance de lecture dans l'abri de jardin. L'activité de jardinage a induit une plus grande diminution du cortisol salivaire (un marqueur du stress) et une plus grande augmentation de l'humeur positive autodéclarée que la séance de lecture (50). Dans la deuxième étude, la perception du corps chez les jardiniers était améliorée après une session de jardinage et les jardiniers avaient une meilleure acceptation de leur corps que les non-jardiniers (44). L'étude longitudinale réalisée à Montpellier n'a pas observé d'effet de la première année de participation à un jardin collectif sur le bien-être psychologique des jardiniers (45).

\section{Jardins collectifs et santé sociale}

Sept études ont évalué la relation entre la santé sociale et la participation à un jardin collectif au travers de divers indicateurs tels que le soutien social perçu (43), l'attachement au quartier (40), la cohésion sociale (48), la solitude (33), l’isolement social (45), le contact avec les amis (33), le capital social (34), les normes et valeurs du quartier (34), le sens de la communauté (32), ou encore l'autonomisation communautaire et organisationnelle (32). Six études sur les sept étaient transversales $(32-34,40,43,48)$. Les données ont été mesurées par questionnaire.

Cinq études transversales sur les six ont fait état d'au moins une association positive entre la participation à un jardin collectif et une variable de santé sociale $(32-34,40,48)$. En population défavorisée, les individus jardinant dans un jardin collectif déclaraient un sentiment plus fort d'appartenance à la communauté et une plus grande autonomisation organisationnelle ou communautaire par rapport à des non-jardiniers (32). Deux études ont montré que les 
jardiniers avaient respectivement des scores de cohésion sociale et d'attachement au quartier plus élevés que les non-jardiniers (40,48). De la même manière, une étude transversale basée sur une enquête représentative en population générale a montré que les adultes appartenant à un ménage dont au moins un membre participait à un jardin collectif ou à un projet d'embellissement de leur quartier avaient des niveaux plus élevés de capital social, de normes et de valeurs de voisinage (telles que l'implication dans le quartier, la satisfaction vis-à-vis du voisinage ou l'efficacité collective) par rapport aux personnes appartenant à un ménage ne participant à aucune de ces activités (34). Une étude a révélé que les personnes de 62 ans et plus fréquentant un jardin collectif se sentaient moins seules que des voisins non-jardiniers du même groupe d'âge, tandis qu'aucune différence significative n'avait été constatée entre les jardiniers et non-jardiniers plus jeunes (33). Dans cette même étude, les jardiniers de 62 ans et plus ont également déclaré avoir des contacts plus fréquents avec leurs amis par rapport aux jardiniers plus jeunes, alors qu'aucune différence de ce type n'a été constatée chez des voisins non jardiniers (33). En revanche, une étude comparant différentes activités de loisir en population âgée n'a pas trouvé de différence de soutien social entre les jardiniers et des personnes participant à d'autres formes d'activité physique (43).

\section{Ancienneté dans le jardin et fréquence du jardinage}

L'ancienneté dans le jardin a été précisée dans six études $(33,39,42,44-46)$. L'étude longitudinale menée sur des jardiniers novices à Montpellier n'a pas observé d'impact de la première année de jardinage sur les variables de santé mesurées (45). Dans les autres études l'ancienneté variait de «moins d'un mois » à « plus de 20 ans ». Seule une étude a évalué l'effet de l'ancienneté de l'appartenance au jardin collectif et n'a pas trouvé d'association entre cette ancienneté et la perception et l'acceptation de son corps (44). 
La fréquence du jardinage a été précisée dans sept études et variait de «tous les jours »

391

392

393

394

395

396

397

398

à «moins d'une fois par mois » $(32,33,36,44,45,47,48)$. Deux études n'ont pas trouvé de relation entre la fréquence de jardinage et les variables de santé étudiées $(45,48)$. A l'inverse, une étude a montré que les jardiniers visitant le jardin quotidiennement déclaraient se sentir plus heureux que ceux s'y rendant moins régulièrement (36). Une autre étude a trouvé que la durée d'une session de jardinage était positivement associée à une meilleure perception du corps chez les jardiniers (44). Le lien entre durée d'exposition au jardin et santé dépendrait en partie des activités réalisées au jardin et des variables de santé étudiées. En effet, une étude a observé une relation positive entre le bien-être et le pourcentage de temps consacré au jardin (incluant le jardinage et les activités d'entretien du jardin), par rapport au temps passé à faire d'autres activités dans le jardin, comme rester assis, lire ou se distraire (33). Dans une autre étude, les jardiniers allant au jardin plus de 12 fois par an déclaraient avoir un plus grand sens de la communauté, tandis que ceux y allant moins souvent déclaraient consommer davantage de légumes (32). Enfin, une dernière étude a estimé la fréquence du jardinage mais ne l'a pas corrélée aux indicateurs de santé mesurés (47).

\section{Évaluation de la qualité méthodologique des études}

Le Tableau 3 présente la qualité des études pour chacun des six domaines d'évaluation de l'EPHPP (biais de sélection, design de l'étude, facteurs de confusion, conduite en aveugle, collecte des données, taux d'attrition). Le risque de biais de sélection était probable dans la plupart des études. Seules trois études, l'une s'appuyant sur une méthode d'échantillonnage aléatoire avec un niveau de participation élevé (34), l'autre sur les données d'enquêtes représentatives en population générale (42) et la dernière recrutant l'ensemble des nouveaux jardiniers intégrant un jardin partagé pour la première fois à Montpellier (45), présentaient un risque modéré de biais de sélection. Conformément aux directives de l' EPHPP, les études 
415 basées sur des échantillons aléatoires mais ayant taux de participation de moins de $60 \%$ $416(35,38,40,41,47)$ et les études basées sur des échantillons de convenance $417(32,33,36,39,43,44,46,48-50)$ ont été jugées de faible qualité. En ce qui concerne le design de

418

l'étude, l'essai contrôlé randomisé (50) a été jugé de bonne qualité ; la quasi-expérimentation (45) et l'étude avant-après une session de jardinage (44) et l'étude post-test uniquement (42) de qualité modérée ; et les autres études de faible qualité en raison de leur conception transversale. Concernant les facteurs de confusion, la plupart des études ont tenu compte des facteurs de confusion potentiels et ce risque était donc considéré comme faible $(n=12)$ (33$35,38,40,41,44,45,47-50)$ ou modéré $(n=2)(32,42)$. En ce qui concerne la conduite en aveugle, quatre études ont utilisé des données provenant d'enquêtes de population et ont été jugées comme étant de qualité modérée parce que les participants ne connaissaient pas la question de recherche $(\mathrm{n}=4)(35,38,40,41)$; les autres études ont été jugées faibles pour ce domaine d'évaluation. En ce qui concerne les méthodes de collecte de données, 12 études ont principalement utilisé des outils dont la validité et fiabilité avaient été préalablement établis $(34,36,38,40,41,43-45,47-50)$ et ont donc été considérées comme étant de bonne qualité concernant ce domaine d'évaluation; les autres études ont été jugées de qualité modérée $(\mathrm{n}=6)(31,32,36,39,40,46)$. Enfin le dernier domaine de l'EPHPP (taux d'attrition) n'était applicable que pour l'essai contrôlé randomisé (50), la quasi-expérimentation (45), l'étude avant-après une session de jardinage (44) et l'étude post-test uniquement (42) qui ont été jugées de bonne qualité. 
Au total, 18 études quantitatives sur la relation entre participation à un jardin collectif urbain et santé en population générale adulte ont été identifiées et incluses dans l'analyse. Une seule étude a été publiée avant 2010 et plus de la moitié au cours des 5 dernières années, montrant un intérêt scientifique croissant pour les jardins collectifs comme outils de promotion de la santé et du bien-être des citadins.

La santé des jardiniers a été évaluée dans ses dimensions physique, mentale et sociale.

Toutes les études transversales ont trouvé au moins une association positive entre la participation à un jardin collectif et un indicateur de santé suggérant que les jardiniers bénéficieraient d'un environnement physique et social, et de conditions psychologiques plus propices à la santé et au bien-être. La fréquence de la consommation de fruits et légumes et l'IMC étaient les variables les plus étudiées. Une association positive a été trouvée entre la participation à un jardin collectif et la consommation de (ou les approvisionnements en) fruits et légumes. Les résultats étaient plus mitigés pour l'IMC et les autres indicateurs de la santé physique. La santé mentale était également très étudiée, et la participation à un jardin collectif était généralement corrélée au bien-être mental et social dans les études transversales. Cette association positive était confortée par les résultats de deux études montrant un effet bénéfique d'une session de jardinage sur la réduction du stress (50) et sur l'amélioration de la perception de son corps (44). Cependant, l'étude Montpelliéraine, qui est la seule étude ayant évalué les changements induits, à plus long terme, par la participation à un jardin partagé, n'a pas trouvé d'effet de la première année de jardinage sur l'alimentation, l'activité physique, l'IMC, le bienêtre mental ni sur l'isolement social (52). Dans cette étude, des entretiens qualitatifs ont révélé les difficultés rencontrées par les nouveaux jardiniers pour s'investir dans le jardin partagé, en particulier le manque de temps, l'absence de connaissances en matière de jardinage, la difficulté physique du jardinage, des problèmes de santé ou encore l'existence de conflits entre jardiniers. 
Ces différents freins avaient d'ailleurs été précédemment identifiés comme des obstacles majeurs à l'implication des jardiniers (53-59).

L'évaluation de la qualité méthodologique des études a souligné leur faiblesse méthodologique. Faciles à mettre en place et peu couteuses, les études observationnelles transversales ne permettent toutefois pas l'inférence causale, il n'est donc pas possible de déterminer si les différences observées sont dues à un biais de sélection. En effet, il se peut que les jardiniers enquêtés dans les études transversales soient justement ceux ayant persévéré dans l'activité de jardinage et ils présenteraient donc des caractéristiques différentes de la population générale, avec notamment une sensibilisation accrue aux enjeux de santé. Or, la causalité est un concept essentiel en santé publique, afin mettre en œuvre des programmes ou des politiques efficaces qui favorisent la santé et le bien-être de la population (60). Une autre limite présente dans la majorité des études existantes est l'évaluation des paramètres de santé par questionnaires auto-déclarés qui sont sujets à des biais de désirabilité et de mémoire (61). A l'exception des deux études ayant évalué objectivement les approvisionnements alimentaires des foyers $(45,46)$, la consommation de fruits et légumes a été estimée par des questionnaires courts dont la validité reste modérée $(62,63)$. Aucune étude n'a utilisé de méthodes rigoureuses d'évaluation des consommations alimentaires telles que les rappels multiples de 24 heures ou des biomarqueurs de la consommation de fruits et légumes. Concernant l'activité physique et la sédentarité, les mesures objectives permettent une évaluation robuste de la dépense énergétique et de l'activité physique de faible intensité (64-67) et doivent donc être privilégiée par rapport aux questionnaires pour évaluer l'impact d'interventions ciblant des modes de vie plus actifs en conditions de vie réelle (67). La seule étude ayant mesuré de façon objective l'activité physique à l'aide d'un accéléromètre était l'étude Montpelliéraine, et elle n'a pas trouvé d'effet de la première année de jardinage sur la dépense énergétique liée à l'activité physique ni sur la sédentarité (45). 
Plusieurs études ont mis en œuvre un design expérimental pour évaluer l'effet de différentes formes de jardinage sur la santé, mais à part une étude dont les résultats n'ont pas encore été publiés (51), aucune d'entre elles n'a porté sur les jardins collectifs urbains et elles ne sont donc pas couvertes par la présente revue de littérature. Les études réalisées sur les activités de jardinage en milieu institutionnalisé (scolaire, médical...) sont néanmoins très instructives. Ainsi, en milieu scolaire, plusieurs interventions de jardinage se sont révélées efficaces pour améliorer la consommation de fruits et légumes des enfants, leurs connaissances et leurs attitudes à l'égard des fruits et légumes, réduire l'IMC et le tour de taille, mais aussi pour améliorer l'activité physique, la pression artérielle ou encore les performances académiques (8). En milieu médical, le jardinage à domicile serait un moyen efficace pour améliorer la consommation de fruits et légumes et les performances physiques de personnes ayant survécu un cancer $(68,69)$ et la thérapie par l'horticulture permettrait d'améliorer la fonction cognitive, l'agitation, les émotions positives ou encore l'engagement dans les activités chez les personnes souffrant de troubles mentaux $(9,10)$.

Plusieurs études réalisées dans des maisons de retraite ont observé un effet positif du jardinage sur la solitude (70), le stress $(71)$, la dépression $(72,73)$ la qualité de vie $(70,72)$, les relations avec autrui $(72,74)$, le sens de la communauté (70), les performances physiques $(71,73)$, ou encore certains marqueurs de maladies chroniques $(74,75)$. Cependant, seule la conduite d'interventions en milieux non-institutionnalisés permettra de déterminer si la participation à un jardin collectif peut favoriser le vieillissement en bonne santé des citadins en limitant les risques de maladies chroniques, de déclin physique, de troubles mentaux et d'isolement social.

Par rapport au jardinage en milieu institutionnel, où l'activité est encadrée et réalisée régulièrement (à hauteur d'au moins une séance par semaine), la fréquence de participation à un jardin collectif est plus variable et dépend principalement du temps libre de chaque individu. 
On peut imaginer que l'effet du jardinage (dans un jardin collectif ou privé) sur la santé dépend en partie de l'exposition au jardin et sera plus important en cas de pratique régulière. Toutefois, le niveau de jardinage requis pour constater un bénéfice sur la santé n'est pas déterminé et les quelques études ayant évalué l'effet sur la santé de la durée ou de la fréquence du jardinage ont conduit à des résultats contradictoires $(32,33,36,44,45,48)$. De nombreuses questions sur le lien entre santé et durée d'exposition au jardin, et plus généralement aux espace verts, restent sans réponses et davantage d'études avec des analyses dose-effet sont nécessaires pour mieux appréhender cette relation (76).

\section{Forces et limites de l'étude}

Cette revue est la première à fournir une évaluation critique des études quantitatives sur le lien entre participation à un jardin collectif urbain et la santé des adultes en population générale. Une des forces de l'analyse est l'utilisation d'une approche systématique basée sur la méthodologie PRISMA. Pour mieux évaluer si les jardins collectifs sont un outil pertinent de promotion de la santé des citadins, nous avons restreint les critères d'inclusion, ce qui a conduit à l'exclusion des études qui n'étaient pas spécifiques des jardins collectifs (77), qui ne distinguaient pas zones rurales et urbaines (78), ou qui n'avaient pas été réalisées dans des zones urbaines telles que définies par le Bureau de recensement des États-Unis (79). Nous avons également exclu de l'analyse les études de type sociologique et/ou anthropologique basées sur des entretiens ou des observations car ces méthodes ne permettent pas de quantifier les relations observées, rendant la comparaison entre les études plus difficile. Enfin, nous avons exclu les études menées dans les pays du Sud où les jardins collectifs sont utilisés comme moyen de subsistance plutôt qu'à des fins récréatives ou éducatives (80). Alors que les programmes de jardinage thérapeutique et scolaire ont prouvé leur efficacité pour améliorer la santé et le bien- 
534 être des participants (6-10), les résultats de la présente revue de littérature montrent que les preuves des bienfaits des jardins collectifs en population générale sont encore limitées.

Une autre force de l'étude est l'évaluation de la qualité méthodologique des études à l'aide de l'outil développé par l'EPHPP. Cet outil a été montré comme étant valide $(81,82)$ et approprié pour évaluer systématiquement des études ne suivant pas un design de type expérimental $(37,83)$. Bien que conçu pour s'adapter une large gamme d'études de santé publique utilisant différents designs, nos résultats suggèrent cependant que l'EPHPP n'est pas l'outil le plus approprié pour évaluer la qualité des études portant sur le jardinage qui ne peuvent être menées à l'aveugle, peuvent difficilement atteindre des taux de participation de plus de $60 \%$, souffrent d'un biais de sélection inhérent (les participants sont de facto intéressés par le jardinage) et évaluent généralement plusieurs paramètres de santé simultanément. La mise en place de critères de jugement plus fins, comme l'utilisation de modalités supplémentaires dans la classification (notamment de seuils additionnels pour le taux de participation), pourrait être un moyen de mieux tenir compte de la complexité des interventions communautaires en prévention et promotions de la santé, et ainsi mieux discriminer les études entre elles.

\section{CONCLUSION ET PISTES DE RECHERCHE}

La littérature sur le lien entre la participation à un jardin collectif et la santé et le bien-être des jardiniers reste limitée. Plusieurs études ont constaté une association positive entre la participation à un jardin partagé et la santé physique, mentale ou sociale, mais les résultats proviennent principalement d'études transversales. Davantage d'études suivant un design expérimental ou quasi-expérimental avec présence d'un groupe contrôle, une taille d'échantillon suffisamment large, des méthodes de mesure validées et analysant la relation dose-effet de l'activité de jardinage, sont nécessaires pour explorer l'effet causal de la participation à un 
558 jardin collectif et de son intensité sur la santé des jardiniers. De telles études permettront de 559 confirmer ou infirmer les bénéfices santé des jardins collectifs suggérés par les études 560 transversales existantes et de déterminer si les jardins collectifs peuvent être un outil efficace 561 pour promouvoir la santé des citadins.

563 Contribution et responsabilité des auteurs : L'ensemble des auteurs attestent du respect des 564 critères de l'International Committee of Medical Journal Editors (ICMJE) en ce qui concerne 565 leur contribution à l'article. M.T et N.D. ont contribué à la conception et à la méthodologie de 566 la présente étude. M.T. a effectué la recherche systématique et a extrait les données. M.T. et N.D. ont procédé à l'évaluation de la qualité méthodologique des

\section{Déclaration d'intérêt : /}

569 Financement : Ce travail a été réalisé dans le cadre de la thèse de MT, qui a bénéficié d'une bourse co-financée par l'INRAE et par le projet Surfood-Foodscape, ce dernier étant financé

571 publiquement par l'Agence Nationale de la Recherche (ANR) dans le cadre du programme

572 "Investissements d'avenir", référence ANR-10-LABX-001-01 Labex Agro, et coordonnés par 573 la Fondation Agropolis. Ce travail a également été soutenu par l'Institut Olga Triballat. Les 574 financeurs n'ont joué aucun rôle dans la conception de l'étude, ni dans la collecte, l'analyse et 575 l'interprétation des données, ni dans la rédaction de l'article. 


\section{Références bibliographiques}

1. WHO Regional Office for Europe. Urban green space interventions and health: A review of impacts and effectiveness. Full report. Copenhagen: Copenhagen: WHO Regional Office for Europe; 2017.

2. WHO Regional Office for Europe. Urban green spaces and health - a review of evidence. Copengague: World Health Organization; 2016.

3. Church A, Mitchell R, Ravenscroft N, Stapleton LM. 'Growing your own': A multi-level modelling approach to understanding personal food growing trends and motivations in Europe. Ecol Econ. 2015;110:71-80.

4. Soga M, Gaston KJ, Yamaura Y. Gardening is beneficial for health: A meta-analysis. Prev Med Reports. 2017;5:92.

5. Wang D, MacMillan T. The Benefits of Gardening for Older Adults: A Systematic Review of the Literature. Act Adapt Aging. 2013;37(2):153-81.

6. Ohly H, Gentry S, Wigglesworth R, Bethel A, Lovell R, Garside R. A systematic review of the health and well-being impacts of school gardening: synthesis of quantitative and qualitative evidence. BMC Public Health. 2016;16(1):286.

7. Savoie-Roskos MR, Wengreen H, Durward C. Increasing Fruit and Vegetable Intake among Children and Youth through Gardening-Based Interventions: A Systematic Review. J Acad Nutr Diet. 2017;117(2):240-50.

8. Rochira A, Tedesco D, Ubiali A, Fantini MP, Gori D. School Gardening Activities Aimed at Obesity Prevention Improve Body Mass Index and Waist Circumference Parameters in SchoolAged Children: A Systematic Review and Meta-Analysis. Child Obes. 2020;16(3):154-73.

9. Zhao Y, Liu Y, Wang Z. Effectiveness of horticultural therapy in people with dementia: A quantitative systematic review. J Clin Nurs. 2020;00:1-15.

10. Cipriani J, Benz A, Holmgren A, Kinter D, McGarry J, Rufino G. A Systematic Review of the Effects of Horticultural Therapy on Persons with Mental Health Conditions. Occup Ther Ment Heal. 2017;33(1):47-69.

11. Kunpeuk W, Spence W, Phulkerd S, Suphanchaimat R, Pitayarangsarit S. The impact of gardening on nutrition and physical health outcomes: a systematic review and meta-analysis. Health Promot Int. 2020;35(2):397-408.

12. Pourias J, Duchemin E, Aubry C. Products from urban collective gardens: Food for thought or for consumption? Insights from Paris and Montreal. J Agric Food Syst Community Dev. 2015;5(2):1-25.

13. Egli V, Oliver M, Tautolo E-S. The development of a model of community garden benefits to wellbeing. Prev Med Reports. 2016;3:348-52.

14. Alaimo K, Beavers AW, Crawford C, Snyder EH, Litt JS. Amplifying health through community gardens: A framework for advancing multicomponent, behaviorally basedneighborhood interventions. Curr Environ Heal Reports. 2016;3(3):302-12.

15. Lawson LJ. City bountiful : a century of community gardening in America. Berkeley and Los Angeles, California: University of California Press; 2005.

16. Kurtz H. Differentiating multiple meanings of garden and community. Urban Geogr. 2001;22(7):656-70.

17. Pourias J, Aubry C, Duchemin E. Is food a motivation for urban gardeners? Multifunctionality and the relative importance of the food function in urban collective gardens of Paris and 
Montreal. Agric Human Values. 2016;33(2):257-73.

18. Crouch D, Ward C. The Allotment: Its Landscape and Culture. Nottingham: Five Leaves Publications; 3rd Revised edition edition; 1997.

19. Archer JE. The Nineteenth-Century Allotment: Half an Acre and a Row. Econ Hist Rev. 1997;50(1):21-36.

20. Draper C, Freedman D. Review and analysis of the benefits, purposes, and motivations associated with community gardening in the United States. J Community Pract. 2010;18(4):458-92.

21. Guitart D, Pickering C, Byrne J. Past results and future directions in urban community gardens research. Urban For Urban Green. 2012;11:364-73.

22. Garcia MT, Ribeiro SM, Germani ACCG, Bógus CM. The impact of urban gardens on adequate and healthy food: a systematic review. Public Health Nutr. 2018

23. Audate PP, Fernandez MA, Cloutier G, Lebel A. Scoping review of the impacts of urban agriculture on the determinants of health. BMC Public Health. 2019

24. Malberg Dyg P, Christensen S, Peterson CJ. Community gardens and wellbeing amongst vulnerable populations: a thematic review. Health Promot Int. 2019;35(4):790-803.

25. McCormack LA, Laska MN, Larson NI, Story M. Review of the Nutritional Implications of Farmers' Markets and Community Gardens: A Call for Evaluation and Research Efforts. J Am Diet Assoc. 2010;110(3):399-408.

26. Machida D, Kushida O. The Influence of Food Production Experience on Dietary Knowledge, Awareness, Behaviors, and Health among Japanese: A Systematic Review. Int J Environ Res Public Health. 2020;17(3):924.

27. Al-Delaimy WK, Webb M. Community Gardens as Environmental Health Interventions: Benefits Versus Potential Risks. Curr Environ Heal Reports. 2017;4(2):252-65.

28. Schram-Bijkerk D, Otte P, Dirven L, Breure AM. Indicators to support healthy urban gardening in urban management. Sci Total Environ. 2018;621:863-71.

29. WHO. Constitution of the World Health Organization. Geneva: World Health Organization; 1948.

30. Liberati A, Altman DG, Tetzlaff J, Mulrow C, Gøtzsche PC, Ioannidis JPA, et al. The PRISMA statement for reporting systematic reviews and meta-analyses of studies that evaluate healthcare interventions: explanation and elaboration. BMJ. 2009;339:b2700.

31. Hall SA, Kaufman JS, Ricketts TC. Defining urban and rural areas in U.S. epidemiologic studies. Vol. 83, Journal of Urban Health. Springer; 2006. p. 162-75.

32. Booth JM, Chapman D, Ohmer ML, Wei K. Examining the Relationship Between Level of Participation in Community Gardens and their Multiple Functions. J Community Pract. 2018;26(1):5-22.

33. Van den Berg AE, Van Winsum-Westra M, De Vries S, Van Dillen SME. Allotment gardening and health: a comparative survey among allotment gardeners and their neighbors without an allotment. Environ Heal. 2010;9(1):74.

34. Alaimo K, Reischl TM, Allen JO. Community gardening, neighborhood meetings, and social capital. J Community Psychol. 2010;38(4):497-514.

35. Litt JS, Lambert JR, Glueck DH. Gardening and age-related weight gain: Results from a crosssectional survey of Denver residents. Prev Med Reports. 2017;8:221-5. 
36. Mourão I, Moreira MC, Almeida TC, Brito LM. Perceived changes in well-being and happiness with gardening in urban organic allotments in Portugal. Int J Sustain Dev World Ecol. 2019;26(1):79-89.

37. Armijo-Olivo S, Stiles CR, Hagen NA, Biondo PD, Cummings GG. Assessment of study quality for systematic reviews: A comparison of the Cochrane Collaboration Risk of Bias Tool and the Effective Public Health Practice Project Quality Assessment Tool: Methodological research. J Eval Clin Pract. 2012;18(1):12-8.

38. Alaimo K, Packnett E, Miles RA, Kruger DJ. Fruit and vegetable intake among urban community gardeners. J Nutr Educ Behav. 2008;40(2):94-101.

39. Algert SJ, Diekmann L, Renvall M, Gray L. Community and home gardens increase vegetable intake and food security of residents in San Jose, California. Calif Agric. 2016;70(2):77-82.

40. Comstock N, Miriam Dickinson L, Marshall JA, Soobader M-J, Turbin MS, Buchenau M, et al. Neighborhood attachment and its correlates: Exploring neighborhood conditions, collective efficacy, and gardening. J Environ Psychol. 2010;30(4):435-42.

41. Litt JS, Soobader M-J, Turbin MS, Hale JW, Buchenau M, Marshall JA. The Influence of Social Involvement, Neighborhood Aesthetics, and Community Garden Participation on Fruit and Vegetable Consumption. Am J Public Health. 2011;101(8):1466.

42. Zick CD, Smith KR, Kowaleski-Jones L, Uno C, Merrill BJ. Harvesting More Than Vegetables: The Potential Weight Control Benefits of Community Gardening. Am J Public Health. 2013;103(6):1110-5.

43. Hawkins JL, Thirlaway KJ, Backx K, Clayton DA. Allotment Gardening and Other Leisure Activities for Stress Reduction and Healthy Aging. Horttechnology. 2011;577-85.

44. Swami V. Body Image Benefits of Allotment Gardening. Ecopsychology. 2020;12(1):19-23.

45. Tharrey M, Sachs A, Perignon M, Simon C, Mejean C, Litt J, et al. Improving lifestyles sustainability through community gardening: results and lessons learnt from the JArDinS quasi-experimental study. BMC Public Health. 2020;20(1):1798.

46. Martin P, Consalès J-N, Scheromm P, Marchand P, Ghestem F, Darmon N. Community gardening in poor neighborhoods in France: A way to re-think food practices? Appetite. 2017;116:589-98.

47. Young C, Hofmann M, Frey D, Moretti M, Bauer N. Psychological restoration in urban gardens related to garden type, biodiversity and garden-related stress. Landsc Urban Plan. 2020;198:103777.

48. Soga M, Cox D, Yamaura Y, Gaston K, Kurisu K, Hanaki K, et al. Health Benefits of Urban Allotment Gardening: Improved Physical and Psychological Well-Being and Social Integration. Int J Environ Res Public Health. 2017;14(1):71.

49. Koay WI, Dillon D. Community gardening: Stress, well-being, and resilience potentials. Int J Environ Res Public Health. 2020;17(18):1-31.

50. Van Den Berg AE, Custers MHG. Gardening promotes neuroendocrine and affective restoration from stress. J Health Psychol. 2011;16(1):3-11.

51. Litt JS, Alaimo K, Buchenau M, Villalobos A, Glueck DH, Crume T, et al. Rationale and design for the community activation for prevention study (CAPs): A randomized controlled trial of community gardening. Contemp Clin Trials. 2018;68:72-8.

52. Tharrey M, Drogué S, Privet L, Perignon M, Dubois C, Darmon N. Industrially processed v. home-prepared dishes: What economic benefit for the consumer? Public Health Nutr. 2020;23(11):1982-90. 
53. Hale J, Knapp C, Bardwell L, Buchenau M, Marshall J, Sancar F, et al. Connecting food environments and health through the relational nature of aesthetics: gaining insight through the community gardening experience. Soc Sci Med. 2011;72(11):1853-63.

54. Lake B, Milfont T, Gavin M. The relative influence of psycho-social factors on urban edible gardening. NZ J Psychol. 2012;41(1):49-58.

55. Wise P. Grow your own: the potential value and impacts of residential and community food gardening. Policy Brief No. 59. Canberra; 2014

56. Niț M-A, Thordin SC. Community gardening initiatives Attitudes and behaviors towards community gardening [Master thesis]. Malmö University; 2020.

57. Kingsley J, Foenander E, Bailey A. "You feel like you're part of something bigger": Exploring motivations for community garden participation in Melbourne, Australia. BMC Public Health. 2019;19(1):745.

58. Loopstra R, Tarasuk V. Perspectives on community gardens, community kitchens and the good food box program in a community-based sample of low-income families. Can J Public Heal. 2013;104(1):e55-9.

59. Diaz JM, Webb ST, Warner LA, Monaghan P. Barriers to community garden success: Demonstrating framework for expert consensus to inform policy and practice. Urban For Urban Green. 2018;31:197-203.

60. Glass TA, Goodman SN, Hernán MA, Samet JM. Causal Inference in Public Health. Annu Rev Public Health. 2013;34(1):61-75.

61. Coughlin SS. Recall bias in epidemiologic studies. J Clin Epidemiol. 1990;43(1):87-91.

62. Kim DJ, Holowaty EJ. Brief, validated survey instruments for the measurement of fruit and vegetable intakes in adults: a review. Prev Med (Baltim). 2003;36(4):440-7.

63. Kristal AR, Vizenor NC, Patterson RE, Neuhouser ML, Shattuck AL, McLerran D. Precision and bias of food frequency-based measures of fruit and vegetable intakes. Cancer Epidemiol Biomarkers Prev. 2000;9(9):939-44.

64. Prince SA, Adamo KB, Hamel M, Hardt J, Connor Gorber S, Tremblay M. A comparison of direct versus self-report measures for assessing physical activity in adults: a systematic review. Int J Behav Nutr Phys Act. 2008;5(1):56.

65. Sylvia LG, Bernstein EE, Hubbard JL, Keating L, Anderson EJ. Practical guide to measuring physical activity. J Acad Nutr Diet. 2014;114(2):199-208.

66. Hills AP, Mokhtar N, Byrne NM. Assessment of physical activity and energy expenditure: an overview of objective measures. Front Nutr. 2014;1(5):1-16.

67. Dowd KP, Szeklicki R, Minetto MA, Murphy MH, Polito A, Ghigo E, et al. A systematic literature review of reviews on techniques for physical activity measurement in adults: a DEDIPAC study. Int J Behav Nutr Phys Act. 2018;15(1):15.

68. Demark-Wahnefried W, Cases MG, Cantor AB, Frugé AD, Smith KP, Locher J, et al. Pilot Randomized Controlled Trial of a Home Vegetable Gardening Intervention among Older Cancer Survivors Shows Feasibility, Satisfaction, and Promise in Improving Vegetable and Fruit Consumption, Reassurance of Worth, and the Trajectory of Central Adiposity. J Acad Nutr Diet. 2018;118(4):689-704.

69. Bail JR, Frugé AD, Cases MG, De Los Santos JF, Locher JL, Smith KP, et al. A home-based mentored vegetable gardening intervention demonstrates feasibility and improvcements in physical activity and performance among breast cancer survivors. Cancer. 2018;124(16):342735 . 
70. Brown VM, Allen AC, Dwozan M, Mercer I, Warren K. Indoor gardening older adults: effects on socialization, activities of daily living, and loneliness. J Gerontol Nurs. 2004;30(10):34-42.

71. Han AR, Park SA, Ahn BE. Reduced stress and improved physical functional ability in elderly with mental health problems following a horticultural therapy program. Complement Ther Med. 2018;38:19-23.

72. Tse MMY. Therapeutic effects of an indoor gardening programme for older people living in nursing homes. J Clin Nurs. 2010;19(7-8):949-58.

73. Park S-A, Lee A-Y, Son K-C, Lee W-L, Kim D-S. Gardening Intervention for Physical and Psychological Health Benefits in Elderly Women at Community Centers. Horttechnology. 2016;26(4):474-483.

74. Ng KST, Sia A, Ng MKW, Tan CTY, Chan HY, Tan CH, et al. Effects of horticultural therapy on asian older adults: A randomized controlled trial. Int J Environ Res Public Health. 2018;15(8):1705.

75. Park S-A, Lee A-Y, Park H-G, Son K-C, Kim D-S, Lee W-L. Gardening Intervention as a Low- to Moderate-Intensity Physical Activity for Improving Blood Lipid Profiles, Blood Pressure, Inflammation, and Oxidative Stress in Women over the Age of 70: A Pilot Study. HortScience. 2017;52(1):200-5.

76. Frumkin H, Bratman GN, Breslow SJ, Cochran B, Kahn PH, Lawler JJ, et al. Nature Contact and Human Health: A Research Agenda. Environ Health Perspect. 2017;125(7):075001(1-18).

77. Litt JS, Schmiege SJ, Hale JW, Buchenau M, Sancar F. Exploring ecological, emotional and social levers of self-rated health for urban gardeners and non-gardeners: A path analysis. Soc Sci Med. 2015;144:1-8.

78. Machida D. Relationship between Community or Home Gardening and Health of the Elderly: A Web-Based Cross-Sectional Survey in Japan. Int J Environ Res Public Health. 2019;16(8):1389.

79. Wood CJ, Pretty J, Griffin M. A case-control study of the health and well-being benefits of allotment gardening. J Public Health (Bangkok). 2016;38(3):e336-44.

80. Eigenbrod C, Gruda N. Urban vegetable for food security in cities. A review. Agron Sustain Dev. 2015;35(2):483-98.

81. Thomas BH, Ciliska D, Dobbins M, Micucci S. A process for systematically reviewing the literature: Providing the research evidence for public health nursing interventions. Worldviews Evidence-Based Nurs. 2004;1(3):176-84.

82. Jackson N, Waters E. Criteria for the systematic review of health promotion and public health interventions. Health Promot Int. 2005;20(4):367-74.

83. Deeks JJ, Dinnes J, D'Amico R, Sowden AJ, Sakarovitch C, Song F, et al. Evaluating nonrandomised intervention studies. Health Technol Assess (Rockv). 2003;7(27):1_173. 


\section{FIGURES}

Figure 1. Diagramme de flux PRISMA 


\section{TABLEAUX}

Tableau 1. Critères PICOS d'inclusion et d'exclusion des études

\begin{tabular}{|c|c|c|}
\hline Paramètres & Critères d'inclusion & Critères d'exclusion \\
\hline Population concernée & $\begin{array}{l}\text { Population générale adulte âgée de } \geq 18 \text { ans } \\
\text { vivant en zone urbaine dans un pays } \\
\text { occidental ou industrialisé }\end{array}$ & $\begin{array}{l}\text { - Population âgée de moins de } 18 \text { ans } \\
\text { - Population non générale en milieu institutionnalisé (hôpital, } \\
\text { maison de repos, centre de santé, prison ou camp de réfugiés) } \\
\text { - Zones rurales } \\
\text { - Pays du sud }\end{array}$ \\
\hline Intervention évaluée & Participation à un jardin collectif & $\begin{array}{l}\text { Jardinage dans un jardin privé, d'ornement, thérapeutique ou } \\
\text { toute autre forme de jardinage ne relevant pas d'un jardin } \\
\text { collectif }\end{array}$ \\
\hline $\begin{array}{l}\text { Comparateur (intervention } \\
\text { servant de comparaison, si } \\
\text { approprié) }\end{array}$ & Non approprié & Non approprié \\
\hline $\begin{array}{l}\text { Outcome(s) (évènement(s) } \\
\text { ou résultat(s) mesuré(s)) }\end{array}$ & $\begin{array}{l}\text { Tout résultat sur la santé physique, mentale } \\
\text { ou sociale issu d'une approche quantitative } \\
\text { (mesures directes ou scores issus de } \\
\text { questionnaires fermés) }\end{array}$ & $\begin{array}{l}\text { - Données issues d'approches non quantitatives (entretiens semi } \\
\text { directifs, questions ouvertes, etc.) } \\
\text { - Données ne relevant pas de la santé }\end{array}$ \\
\hline
\end{tabular}


Analyses documentaires, études de cas, thèses, mémoires,

interventionnelles utilisant une approche

lettres à l'éditeur, chapitres de livres et commentaires d'auteurs quantitative 
Tableau 2. Caractéristiques des études quantitatives sur le lien entre jardin collectif urbain et santé en population générale adulte ${ }^{a}$

\begin{tabular}{|c|c|c|c|c|c|c|c|}
\hline Référence & Lieu & $\begin{array}{l}\text { Design de } \\
\text { l'étude }\end{array}$ & $\begin{array}{c}\text { Population, } \\
\text { Groupes d'étude (G1, } \\
\text { G2...) } \\
\end{array}$ & $\begin{array}{l}\text { Age moyen, } \\
\text { \% Femmes }\end{array}$ & $\begin{array}{c}\text { Durée et } \\
\text { fréquence de } \\
\text { jardinage }\end{array}$ & $\begin{array}{l}\text { Données collectées } \\
\text { (Outils d'évaluation) }\end{array}$ & Principaux résultats $^{b}$ \\
\hline $\begin{array}{l}\text { Alaimo et } \\
\text { al. } \\
(2008)(38)\end{array}$ & $\begin{array}{l}\text { Flint, MI, } \\
\text { USA }\end{array}$ & $\begin{array}{l}\text { Transversal, } \\
\text { échantillon } \\
\text { aléatoire } \\
\text { représentatif de } \\
\text { la population } \\
\text { générale, GC }\end{array}$ & $\begin{array}{l}\mathrm{n}=766 \text { foyers avec : } \\
\mathrm{G} 1: \text { au moins } 1 \text { membre } \\
\text { participant un jardin partagé } \\
(\mathrm{n}=116) \\
\mathrm{G} 2: \text { aucun membre } \\
\text { participant un jardin partagé } \\
(\mathrm{n}=650)\end{array}$ & $\begin{array}{l}43,8 \text { ans } \\
51,9 \% \mathrm{~F}\end{array}$ & NS & $\begin{array}{l}\text { Alimentation: } \\
\text { Consommation de fruits et légumes (BRFSS) }\end{array}$ & $\begin{array}{l}\text { G1 consomme des fruits et } \\
\text { légumes } 1,4 \text { fois plus que } \mathrm{G} 2 \text {, } \\
\text { et est } 3,5 \text { fois plus susceptible } \\
\text { de consommer des fruits et } \\
\text { légumes au moins } 5 \text { fois/j. }\end{array}$ \\
\hline $\begin{array}{l}\text { Alaimo et } \\
\text { al. } \\
(2010)(34)\end{array}$ & $\begin{array}{l}\text { Flint, MI, } \\
\text { USA }\end{array}$ & $\begin{array}{l}\text { Transversal, } \\
\text { échantillon } \\
\text { aléatoire } \\
\text { représentatif de } \\
\text { la population } \\
\text { générale, GC }\end{array}$ & $\begin{array}{l}\mathrm{n}=1916 \text { foyers participant à : } \\
\mathrm{G} 1: \text { jardin partagé ou projet } \\
\text { d'embellissement }(\mathrm{n}=271) \\
\text { G2: associations de quartiers } \\
(\mathrm{n}=129) \\
\text { G3: G1 \& G2 }(\mathrm{n}=292) \\
\text { G4: ni G1 ni G2 } \\
(\mathrm{n}=1224)\end{array}$ & $\begin{array}{l}\mathrm{G} 1: 40,7 \text { ans, } \\
54.3 \% \mathrm{~F} \\
\mathrm{G} 2: 45,9 \text { ans, } \\
47.7 \% \mathrm{~F} \\
\mathrm{G} 3: 43,8 \text { ans, } \\
47,7 \% \mathrm{~F} \\
\mathrm{G} 4: 45,5 \text { ans, } \\
56,7 \% \mathrm{~F}\end{array}$ & NS & \begin{tabular}{|l} 
Santé sociale: \\
- Capital social qui unit (bonding) (12 items) \\
- Capital social qui lie (linking) (3 items) \\
- Normes et valeurs du voisinage (14 items)
\end{tabular} & $\begin{array}{l}\text { Capital social et normes et } \\
\text { valeurs du voisinage plus } \\
\text { élevés dans G1, G2 et G3 que } \\
\text { dans G4. Les associations } \\
\text { étaient plus élevées pour G3 } \\
\text { que G1 ou G2. }\end{array}$ \\
\hline $\begin{array}{l}\text { Algert et al. } \\
(2016)(39)\end{array}$ & $\begin{array}{c}\text { San Jose, CA, } \\
\text { USA }\end{array}$ & $\begin{array}{l}\text { Transversal, } \\
\text { échantillon de } \\
\text { convenance, GC }\end{array}$ & $\begin{array}{l}\mathrm{n}=135 \text { adultes, population } \\
\text { défavorisée: } \\
\mathrm{G} 1: \text { jardiniers dans des } \\
\text { jardins privé }(\mathrm{n}=50) \\
\mathrm{G} 2: \text { jardiniers dans des } \\
\text { jardins partagés }(\mathrm{n}=85)\end{array}$ & $\begin{array}{l}\text { G1: } 49 \text { ans, } \\
84 \% \mathrm{~F} \\
\text { G2: } 58 \text { ans, } \\
50 \% \mathrm{~F}\end{array}$ & $\begin{array}{l}\text { Durée : } \\
\text { G1: } 48 \% \text { avec < } \\
2 \text { ans } \\
\text { d'expérience } \\
\text { G2: } 33 \% \text { avec < } \\
2 \text { ans } \\
\text { d'expérience }\end{array}$ & $\begin{array}{l}\text { Alimentation: } \\
\text { Consommation de légumes } \\
\text { (EFNEP food behaviour checklist) } \\
\text { Anthropométrie : } \\
\text { IMC (poids et taille déclarés) }\end{array}$ & $\begin{array}{l}\text { G1 et G2 ont déclaré avoir } \\
\text { doublé leur consommation de } \\
\text { légumes, et atteignaient un } \\
\text { niveau conforme aux } 2,5 \\
\text { portions quotidiennes } \\
\text { recommandées aux États- } \\
\text { Unis (G1 : }+1,9 \text { tasses } / \mathrm{j} ; \mathrm{G} 2 \text { : } \\
+2,0 \text { tasses } / \mathrm{j}) . \text { Pas de } \\
\text { différence d'IMC entre les } \\
\text { deux groupes. }\end{array}$ \\
\hline
\end{tabular}




\begin{tabular}{|c|c|c|c|c|c|c|c|}
\hline \begin{tabular}{|l|}
$\begin{array}{l}\text { Booth et al., } \\
(2018)(32)\end{array}$ \\
\end{tabular} & $\begin{array}{l}\text { USA } \\
\text { Zones } \\
\text { urbaines }\end{array}$ & $\begin{array}{l}\text { Transversal, } \\
\text { échantillon de } \\
\text { convenance, GC }\end{array}$ & $\begin{array}{l}\mathrm{n}=115 \text { adultes, population } \\
\text { défavorisée: } \\
\text { G1: jardiniers réguliers dans } \\
\text { des jardins partagés }(\mathrm{n}=16) \\
\text { G2: jardiniers occasionnels } \\
\text { dans des jardins partagés }(\mathrm{n}= \\
\text { 43) } \\
\text { G3: non-jardiniers }(\mathrm{n}=56)\end{array}$ & $\begin{array}{l}42,1 \text { ans, } \\
57,8 \% \mathrm{~F}\end{array}$ & $\begin{array}{l}\text { Participation : } \\
\cdot \text { jardiniers actifs } \\
(>12 \text { visites/an }) \\
(\mathrm{n}=16) \\
\cdot \text { jardiniers } \\
\text { occasionnels } \\
(\leq 12 \text { visites/an }) \\
(\mathrm{n}=43)\end{array}$ & 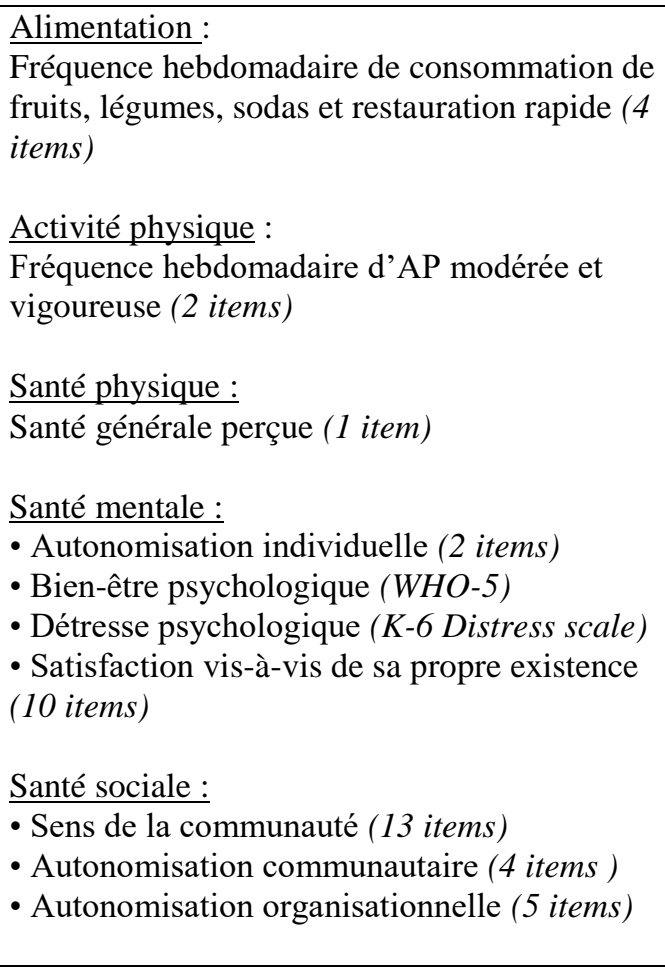 & $\begin{array}{l}\text { Bien-être psychologique et } \\
\text { autonomisation } \\
\text { communautaire plus élevés } \\
\text { dans G1 et G2 que dans G3. } \\
\text { Autonomisation } \\
\text { organisationnelle plus élevée } \\
\text { dans G1 que dans G2 et G3 et } \\
\text { sens de la communauté plus } \\
\text { élevé dans G1 que dans G2. } \\
\text { Le G2 consomme des } \\
\text { légumes } 1,3 \text { fois de plus par } \\
\text { semaine et a un score de } \\
\text { détresse psychologique } \\
\text { inférieur au G3. } \\
\text { Pas de différence de } \\
\text { fréquence de consommation } \\
\text { de fruits, de sodas et de } \\
\text { restauration rapide, d'activité } \\
\text { physique, de santé générale, } \\
\text { d'autonomisation et de } \\
\text { satisfaction vis-à-vis de sa } \\
\text { propre existence entre les } \\
\text { groupes }\end{array}$ \\
\hline $\begin{array}{l}\text { Comstock } \\
\text { et al. } \\
(2010)(40)\end{array}$ & $\begin{array}{l}\text { Denver, CO, } \\
\text { USA }\end{array}$ & $\begin{array}{l}\text { Transversal, } \\
\text { échantillon } \\
\text { aléatoire de type } \\
\text { multi-stage }{ }^{c}, \mathrm{GC}\end{array}$ & $\begin{array}{l}\mathrm{n}=410 \text { adultes: } \\
\text { G1: jardiniers dans des } \\
\text { jardins partagés }(\mathrm{n}=31) \\
\text { G2: jardiniers dans des } \\
\text { jardins privés }(\mathrm{n}=197) \\
\text { G3: } \text { non-jardiniers }(\mathrm{n}=182) \\
\end{array}$ & $\begin{array}{l}\text { Âge médian : } \\
45 \text { ans, } \\
\text { (écart : 18-94) } \\
\text { NA }\end{array}$ & NS & $\begin{array}{l}\text { Santé sociale : } \\
\text { Attachement individuel au quartier (6 items) }\end{array}$ & $\begin{array}{l}\text { Plus grand attachement } \\
\text { individuel au quartier dans } \\
\text { G1 et G2 que dans G3. }\end{array}$ \\
\hline
\end{tabular}




\begin{tabular}{|c|c|c|c|c|c|c|c|}
\hline $\begin{array}{l}\text { Hawkins et } \\
\text { al. } \\
(2011)(43)\end{array}$ & $\begin{array}{c}\text { Cardiff, } \\
\text { Royaume-Uni }\end{array}$ & $\begin{array}{l}\text { Transversal, } \\
\text { échantillon de } \\
\text { convenance, GC }\end{array}$ & $\begin{array}{l}\mathrm{n}=94 \text { adultes }>50 \text { ans: } \\
\text { G1: jardiniers dans des } \\
\text { jardins familiaux }(\mathrm{n}=25) \\
\text { G2: jardiniers dans des } \\
\text { jardins privés }(\mathrm{n}=21) \\
\text { G3: membres de groupes de } \\
\text { marche }(\mathrm{n}=25) \\
\text { G4: membres de groupes } \\
\text { d'AP en salle }(\mathrm{n}=23)\end{array}$ & $\begin{array}{l}\text { G1: } 65,7 \text { ans, } \\
8 \% \mathrm{~F} \\
\text { G2: } 69,5 \text { ans, } \\
19 \% \mathrm{~F} \\
\text { G3: } 62,4 \text { ans, } \\
17 \% \mathrm{~F} \\
\text { G4: } 72,9 \text { ans, } \\
20 \% \mathrm{~F}\end{array}$ & NS & $\begin{array}{l}\text { Anthropométrie : } \\
\text { IMC (mesures objectives) } \\
\text { Activité physique : } \\
\text { - Fréquence et durée de l'AP modérée et } \\
\text { vigoureuse (min/semaine) (IPAQ version } \\
\text { courte) } \\
\text { - Temps passé assis (min/semaine) (IPAQ } \\
\text { version courte) } \\
\text { Santé physique : } \\
\text { - Pression artérielle } \\
\text { - Fonction pulmonaire • Santé physique } \\
\text { générale (Quality of Life } Q \text { (SF-36v2)) } \\
\text { Santé mentale : } \\
\text { - Stress perçu (Perceived stress scale) } \\
\text { - Santé mentale générale (Quality of Life } Q \\
\text { (SF-36v2)) } \\
\text { Santé sociale : } \\
\text { Support social perçu (Social provisions scale) }\end{array}$ & $\begin{array}{l}\text { Niveau de stress plus faible } \\
\text { dans G1 que dans G4 }(9,8 \pm \\
5,8 \text { vs } 15,8 \pm 6,1) \text {. Pas de } \\
\text { différence entre les groupes } \\
\text { pour les autres variables. }\end{array}$ \\
\hline $\begin{array}{l}\text { Koay et al. } \\
(2020)(49)\end{array}$ & Singapour & $\begin{array}{l}\text { Transversal, } \\
\text { échantillon de } \\
\text { convenance par } \\
\text { boule de neige, } \\
\text { GC }\end{array}$ & $\begin{array}{l}\mathrm{n}=111 \text { adultes: } \\
\mathrm{G} 1: \text { jardiniers dans des } \\
\text { jardins partagés }(\mathrm{n}=45) \\
\mathrm{G} 2: \text { jardiniers dans des } \\
\text { jardins privés }(\mathrm{n}=38) \\
\mathrm{G} 3: \text { non-jardiniers engagés } \\
\text { dans des AP de plein air }(\mathrm{n}= \\
\text { 28) }\end{array}$ & $\begin{array}{l}\mathrm{G} 1: 60,2 \text { ans, } \\
44,4 \% \mathrm{~F} \\
\text { G2: } 43,8 \text { ans, } \\
84,2 \% \mathrm{~F} \\
\text { G3: } 55,5 \text { ans, } \\
57,1 \% \mathrm{~F}\end{array}$ & NS & $\begin{array}{l}\text { Santé mentale : } \\
\text { - Bien-être subjectif (Personal Wellbeing } \\
\text { Index-Adult) } \\
\text { - Stress (Perceived Stress Scale) } \\
\text { - Résilience (Brief Resilience Scale) } \\
\text { - Estime de soi (Rosenberg Self-Esteem Scale) } \\
\text { - Optimisme (Life Orientation Test-Revised) } \\
\text { - Ouverture (Openness-to- Experience scale } \\
\text { from the IPIP) }\end{array}$ & $\begin{array}{l}\text { Bien-être subjectif plus élevé } \\
\text { dans G1 que dans G2 et G3 } \\
(8,2,7,0 \text { et } 7,0 \\
\text { respectivement). Par rapport } \\
\text { au } G 3, G 1 \text { a un score plus } \\
\text { élevé de résilience }(3,7 \text { vs } \\
3,0) \text { et d'optimisme ( } 16,8 \text { vs } \\
13,8) \text {. Pas de différence entre } \\
\text { les groupes pour les autres } \\
\text { variables. }\end{array}$ \\
\hline \begin{tabular}{|l|} 
Litt et al. \\
$(2011)(41)$
\end{tabular} & $\begin{array}{c}\text { Denver, CO, } \\
\text { USA }\end{array}$ & $\begin{array}{l}\text { Transversal, } \\
\text { échantillon } \\
\text { aléatoire de type } \\
\text { multi-stagec }, \text { GC }\end{array}$ & $\begin{array}{l}\mathrm{n}=436 \text { adultes: } \\
\text { G1: jardiniers dans des } \\
\text { jardins partagés }(\mathrm{n}=41) \\
\text { G2: jardiniers dans des } \\
\text { jardins privés }(\mathrm{n}=208) \\
\mathrm{G} 3 \text { : non jardiniers }(\mathrm{n}=187)\end{array}$ & $\begin{array}{l}46 \text { ans, } \\
68 \% \mathrm{~F}\end{array}$ & NS & $\begin{array}{l}\text { Alimentation: } \\
\text { Consommation de fruits et légumes (BRFSS) }\end{array}$ & $\begin{array}{l}\text { Plus grande fréquence de } \\
\text { consommation de fruits et } \\
\text { légumes dans G1 que dans } \\
\text { G2 et G3 (5,0, } 4,3 \text { et } 3,9 \\
\text { fois/jour respectivement) }\end{array}$ \\
\hline
\end{tabular}




\begin{tabular}{|c|c|c|c|c|c|c|c|}
\hline $\begin{array}{l}\text { Litt et al. } \\
(2017)(35)\end{array}$ & $\begin{array}{l}\text { Denver, CO, } \\
\text { USA }\end{array}$ & $\begin{array}{l}\text { Transversal, } \\
\text { échantillon } \\
\text { aléatoire de type } \\
\text { multi-stagec }, \text { GC }\end{array}$ & $\begin{array}{l}\mathrm{n}=469 \text { adultes: } \\
\text { G1: jardiniers dans des } \\
\text { jardins partagés }(\mathrm{n}=63) \\
\text { G2: jardiniers dans des } \\
\text { jardins privés }(\mathrm{n}=215) \\
\text { G3: non jardiniers }(\mathrm{n}=191)\end{array}$ & $\begin{array}{l}46,1 \text { ans } \\
67,4 \% \mathrm{~F}\end{array}$ & NS & \begin{tabular}{|l} 
Anthropométrie : \\
IMC (poids et taille déclarés)
\end{tabular} & $\begin{array}{l}\text { Les participants du G1 et G2 } \\
\text { ont un IMC inférieur à ceux } \\
\text { du G3. Augmentation de } \\
\text { l'IMC de } 0,03 \mathrm{~kg} / \mathrm{m}^{2} \text { pour } \\
\text { chaque année d'augmentation } \\
\text { de l'âge uniquement dans G3, } \\
\text { mais l'association disparait } \\
\text { après ajustement. }\end{array}$ \\
\hline $\begin{array}{l}\text { Martin et al. } \\
(2017)(46)\end{array}$ & $\begin{array}{l}\text { Marseille, } \\
\text { France }\end{array}$ & $\begin{array}{l}\text { Transversal, } \\
\text { échantillon de } \\
\text { convenance, GC }\end{array}$ & $\begin{array}{l}\mathrm{n}=87 \text { adultes, population } \\
\text { défavorisée : } \\
\text { G1: jardiniers dans des } \\
\text { jardins partagés }(\mathrm{n}=21) \\
\text { G2: non jardiniers }(\mathrm{n}=65)\end{array}$ & $\begin{array}{l}\text { G1: } 52,0 \text { ans, } \\
100 \% \mathrm{~F} \\
\text { G2: NS } 100 \% \\
\text { F }\end{array}$ & $\begin{array}{l}\text { Durée moyenne } \\
\text { d'occupation du } \\
\text { jardin : } 21 \text { mois } \\
\text { (écart : } 0-48 \\
\text { mois) }\end{array}$ & $\begin{array}{l}\text { Alimentation: } \\
\text { Approvisionnement alimentaire des foyers } \\
\text { (achats, dons et récoltes du jardin) (carnet des } \\
\text { approvisionnements alimentaires) }\end{array}$ & $\begin{array}{l}\text { Approvisionnements plus } \\
\text { élevés en fruits et légumes } \\
\text { dans G1 que dans G2 }(+158 \\
\text { g/j.pers). Pas de différences } \\
\text { pour les autres groupes } \\
\text { alimentaires }\end{array}$ \\
\hline $\begin{array}{l}\text { Mourão et } \\
\text { al. } \\
(2018)(36)\end{array}$ & $\begin{array}{l}\text { Vila Nova de } \\
\text { Famalicão, } \\
\text { Portugal }\end{array}$ & $\begin{array}{l}\text { Transversal, } \\
\text { échantillon de } \\
\text { convenance }\end{array}$ & $\begin{array}{l}\mathrm{n}=65 \text { jardiniers dans des } \\
\text { jardins familiaux }\end{array}$ & $\begin{array}{l}\cdot 25-45 \text { ans : } \\
37 \% \\
\cdot 46-65 \text { ans : } \\
48 \% \\
\bullet>65 \text { ans : } \\
15 \% \\
43,1 \% \mathrm{~F}\end{array}$ & \begin{tabular}{|l|} 
Jardinant : \\
$\cdot$ tous les jours \\
$(41.5 \%)$ \\
$\cdot$ plusieurs \\
fois/semaine $(\mathrm{n}=$ \\
$47.7 \%)$ \\
$\cdot$ une fois par \\
semaine $(10.8 \%)$ \\
\end{tabular} & $\begin{array}{l}\text { Santé mentale: } \\
\text { Bonheur subjectif } \\
\text { (subjective happiness scale) }\end{array}$ & $\begin{array}{l}\text { Bonheur subjectif plus élevé } \\
\text { chez les jardiniers visitant le } \\
\text { jardin tous les jours par } \\
\text { rapport à leurs pairs. }\end{array}$ \\
\hline $\begin{array}{l}\text { Soga et al. } \\
(2017)(48)\end{array}$ & Tokyo, Japon & $\begin{array}{l}\text { Transversal, } \\
\text { échantillon de } \\
\text { convenance, GC }\end{array}$ & $\begin{array}{l}\mathrm{n}=332 \text { adultes: } \\
\text { G1: jardiniers dans des } \\
\text { jardins familiaux }(\mathrm{n}=165) \\
\text { G2: non jardiniers }(\mathrm{n}=167)\end{array}$ & $\begin{array}{l}\text { G1: } 61,9 \text { ans, } \\
31,9 \% \mathrm{~F} \\
\text { G2: } 61,0 \text { ans, } \\
58,2 \% \mathrm{~F}\end{array}$ & \begin{tabular}{|l|} 
Nombre moyen \\
de visites : $15,7 \pm$ \\
10,9 fois/mois \\
Durée moyenne \\
de chaque visite : \\
$80,0 \pm 64,9$ min \\
Durée moyenne \\
totale de toutes \\
les visites $: 21,0$ \\
$\pm 25,7 \mathrm{~h} /$ mois \\
\end{tabular} & $\begin{array}{l}\text { Anthropométrie : } \\
\text { IMC (poids et taille déclarés) } \\
\text { Santé physique : } \\
\text { - Santé générale perçue ( } 1 \text { item) } \\
\text { - Problèmes de santé déclarés (10 items) } \\
\text { Santé mentale : } \\
\begin{array}{l}\text { Détresse psychologique / troubles de l'humeur } \\
\text { (General Health } Q \text { ) }\end{array} \\
\text { Santé sociale : } \\
\text { Cohésion sociale (Social Cohesion and Trust } \\
\text { Scale) }\end{array}$ & $\begin{array}{l}\text { Santé générale et cohésion } \\
\text { sociale plus élevées dans G1 } \\
\text { que dans G2. Problèmes de } \\
\text { santé déclarés et détresse } \\
\text { psychologique plus faibles } \\
\text { dans G1 que dans G2. } \\
\text { Pas de différence d'IMC entre } \\
\text { les deux groupes. }\end{array}$ \\
\hline
\end{tabular}




\begin{tabular}{|c|c|c|c|c|c|c|c|}
\hline $\begin{array}{l}\text { Swami et al. } \\
(2020)(44)\end{array}$ & $\begin{array}{l}\text { London, } \\
\text { Royaume-Uni }\end{array}$ & $\begin{array}{l}\text { Etude avant- } \\
\text { après, échantillon } \\
\text { de convenance, } \\
\text { mesure avant- } \\
\text { près (une séance } \\
\text { de jardinage) } \\
\text { uniquement chez } \\
\text { les jardiniers, CG }\end{array}$ & $\begin{array}{l}\mathrm{n}=165 \text { adultes: } \\
\text { G1: jardiniers dans des } \\
\text { jardins familiaux }(\mathrm{n}=84) \\
\text { G2: non jardiniers appariés }(\mathrm{n} \\
=81)\end{array}$ & $\begin{array}{l}44,7 \text { ans, } \\
60 \% \mathrm{~F}\end{array}$ & $\begin{array}{l}\text { Durée moyenne } \\
\text { d'occupation du } \\
\text { jardin : } 58 \text { mois } \\
\text { Durée moyenne } \\
\text { d'une session de } \\
\text { jardinage }: 2,5 \mathrm{~h}\end{array}$ & \begin{tabular}{|l} 
Santé mentale: \\
- Perception du corps (Visual analogue scale) \\
- Acceptation de son corps (Body Appreciation \\
Scale-2) \\
- Appréciation de la fonctionnalité du corps \\
(Functionality Appreciation Scale) \\
- Fierté du corps (Authentic Pride subscale of \\
the Body and Appearance Self-Conscious \\
Emotions Scale)
\end{tabular} & $\begin{array}{l}\text { Amélioration du score de } \\
\text { perception du corps du G1 } \\
\text { avant et après une session de } \\
\text { jardinage }(62,6 \text { vs } 53,0) \text {. } \\
\text { Par rapport au G2, G1 a une } \\
\text { meilleure acceptation de son } \\
\text { corps }(3,5 \text { vs } 3,1) \text {, } \\
\text { appréciation de la } \\
\text { fonctionnalité du corps }(3,5 \\
\text { vs } 3,2) \text { et }(3,1 \text { vs } 2,6) \text {. }\end{array}$ \\
\hline $\begin{array}{l}\text { Tharrey et } \\
\text { al.(2020)(45 } \\
\text { ) }\end{array}$ & $\begin{array}{l}\text { Montpellier, } \\
\text { France }\end{array}$ & \begin{tabular}{|l|} 
Etude quasi- \\
expérimentale, \\
tous les \\
nouveaux \\
jardiniers, \\
mesure avant- \\
près (une année \\
de jardinage), \\
GC
\end{tabular} & $\begin{array}{l}\mathrm{n}=132 \text { adultes: } \\
\text { G1: jardiniers dans des } \\
\text { jardins partagés }(\mathrm{n}=66) \\
\text { G2: non jardiniers appariés (n } \\
=66)\end{array}$ & $\begin{array}{l}\mathrm{G} 1: 44 \text { ans, } \\
75,8 \% \mathrm{~F} \\
\mathrm{G} 2: 44,9 \text { ans, } \\
75,8 \% \mathrm{~F}\end{array}$ & $\begin{array}{l}\text { Débutants dans } \\
\text { un jardin partagé. } \\
\text { Au moins } 1 \\
\text { fois/mois : } \\
\bullet \text { Toute l'année } \\
(56 \%) \\
\bullet[6-9[\text { mois } \\
(18 \%) \\
\bullet[3-6[\text { mois } \\
(17 \%), \\
\bullet<3 \text { mois }(9 \\
\%) .\end{array}$ & 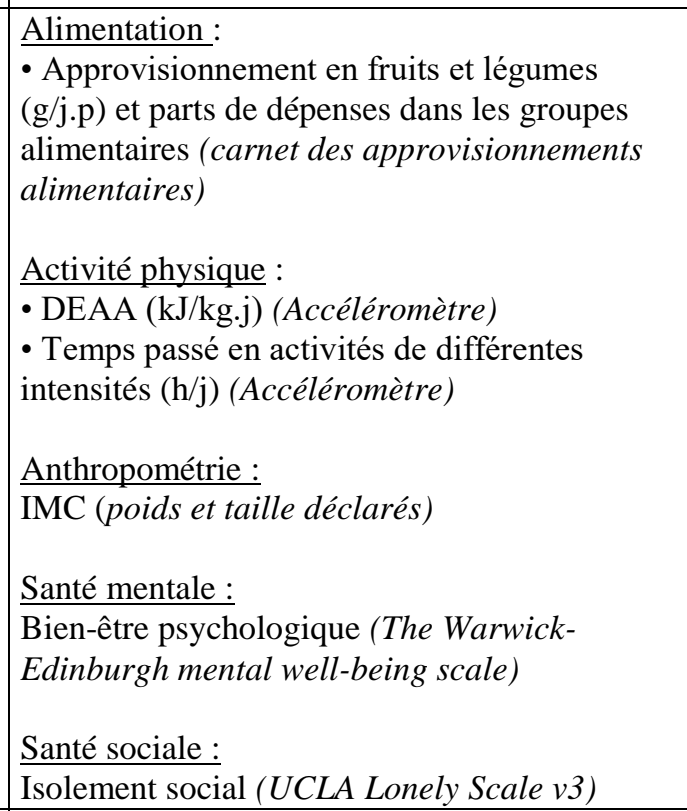 & $\begin{array}{l}\text { Pas de d'évolution des } \\
\text { différentes variables après } 1 \\
\text { an dans le jardin. }\end{array}$ \\
\hline $\begin{array}{l}\text { Van den } \\
\text { Berg et al. } \\
(2010)(33)\end{array}$ & $\begin{array}{c}\text { Pays-Bas } \\
\text { Urbain }(65 \%) \\
\text { Péri-urbain } \\
(31 \%) \\
\text { Rural }(4 \%)\end{array}$ & $\begin{array}{l}\text { Transversal, } \\
\text { échantillon de } \\
\text { convenance, GC }\end{array}$ & $\begin{array}{l}\mathrm{n}=184 \text { adultes: } \\
\text { G1: jardiniers dans des } \\
\text { jardins familiaux } \geq 62 \text { ans ( } \\
=70 \text { ) } \\
\text { G2: } \mathrm{G} 1: \text { jardiniers dans des } \\
\text { jardins familiaux }<62 \text { ans ( } \mathrm{n} \\
=51) \\
\text { G3: voisins non jardiniers } \\
\geq 62 \text { ans }(\mathrm{n}=21)\end{array}$ & $\begin{array}{l}\text { G1: } 61,5 \text { ans, } \\
47 \% \mathrm{~F} \\
\text { G2: } 55,9 \text { ans, } \\
59 \% \mathrm{~F}\end{array}$ & \begin{tabular}{|l} 
Durée du \\
jardinage : \\
$\bullet$ G1 :11-20 ans \\
$\bullet$ G2: $6-10$ ans \\
\\
$\begin{array}{l}\text { Temps moyen } \\
\text { consacré aux } \\
\text { activités de } \\
\text { jardinage et }\end{array}$ \\
\end{tabular} & $\begin{array}{l}\text { Activité physique : } \\
\text { Fréquence d'AP en été et hiver (2 items du } \\
\text { SQUASH) } \\
\text { Santé physique : } \\
\text { - Santé générale perçue (1 item) } \\
\text { - Contrainte physique (Physical functioning } \\
\text { subscale - SF-36) } \\
\text { - Problèmes de santé déclarés ( } 7 \text { items) }\end{array}$ & $\begin{array}{l}\text { Fréquence d'AP plus élevée } \\
\text { pendant l'été dans } G 1 \text { et } G 2 \\
\text { que dans } \mathrm{G} 3 \text { et } \mathrm{G} 4(5,8 \pm \\
1,53 \text { vs } 4,9 \pm 2,15 \mathrm{j} / \mathrm{semaine}) \text {. } \\
\text { Par rapport au } \mathrm{G} 3 \text {, G1 déclare } \\
\text { moins de contraintes } \\
\text { physiques }(1,27 \pm 0,04 \text { vs } \\
1,53 \pm 0,07) \text {, de problèmes de } \\
\text { santé déclarés }(2,04 \pm 0,26 \text { vs }\end{array}$ \\
\hline
\end{tabular}




\begin{tabular}{|c|c|c|c|c|c|c|c|}
\hline & & & $\begin{array}{l}\text { G4 : voisins non jardiniers } \\
<62 \text { ans }(\mathrm{n}=42)\end{array}$ & & $\begin{array}{l}\text { d'entretien du } \\
\text { jardin : } \\
\text { • G1: } 66 \% \\
\text { - G2: } 56 \%\end{array}$ & $\begin{array}{l}\text { - Maladies chroniques }(5 \text { items) } \\
\text { - Consultations chez le médecin (1 item) } \\
\text { Santé mentale : } \\
\text { - Stress (2 items) } \\
\text { - Satisfaction vis-à-vis de sa propre existence } \\
\text { (life Satisfaction Index) } \\
\text { - Solitude (2 items) } \\
\text { Santé sociale : } \\
\text { Contact social avec les amis (2 items) }\end{array}$ & $\begin{array}{l}3,83 \pm 0,45) \text {, de consultations } \\
\text { chez le médecin }(0,52 \pm 0,13 \\
\text { vs } 1,14 \pm 0,23) \text { et de solitude } \\
(0,28 \pm 0,09 \text { contre } 0,8 \pm \\
0,16) \text { ainsi qu'une plus grande } \\
\text { satisfaction vis-à-vis de sa } \\
\text { propre existence }(2,29 \pm 0,06 \\
\text { vs } 1,96 \pm 0,09) \text {. Par rapport } \\
\text { au G2, G1 déclare un stress } \\
\text { plus faible }(2,05 \pm 1,2 \text { vs } 3,20 \\
\pm 0,14) \text { et plus de contacts } \\
\text { sociaux }(8,07 \pm 0,38 \text { vs } 6,14 \\
\pm 0,43) \text {, alors qu'il n'y a pas } \\
\text { de différence entre G2 \& G4. } \\
\text { Pas de différence entre les } \\
\text { groupes en ce qui concerne la } \\
\text { santé générale et les maladies } \\
\text { chroniques. }\end{array}$ \\
\hline $\begin{array}{l}\text { Van den } \\
\text { Berg et al. } \\
\text { (2011)(50) }\end{array}$ & $\begin{array}{l}\text { Amsterdam, } \\
\text { Pays-Bas }\end{array}$ & $\begin{array}{l}\text { Essai contrôlé } \\
\text { randomisé }\end{array}$ & $\begin{array}{l}\mathrm{n}=30 \text { jardiniers dans des } \\
\text { jardins familiaux. } \\
\text { Après avoir effectué une } \\
\text { tâche stressante, les } \\
\text { participants ont été } \\
\text { randomisés pour } 30 \text { min de : } \\
\mathrm{G} 1: \text { activités de jardinage en } \\
\text { plein air }(\mathrm{n}=14) \\
\mathrm{G} 2: \text { lecture à l'intérieur }(\mathrm{n}= \\
\text { 16) }\end{array}$ & $\begin{array}{l}\text { G1: } 58,3 \text { ans, } \\
72 \% \mathrm{~F} \\
\mathrm{G} 2: 57,0 \text { ans } \\
75 \% \mathrm{~F}\end{array}$ & $\mathrm{~N} / \mathrm{S}$ & $\begin{array}{l}\text { Santé mentale: } \\
\text { - Cortisol salivaire (prélèvement) } \\
\text { - Humeur (Positive and Negative Affect } \\
\text { Schedule) }\end{array}$ & $\begin{array}{l}\text { Après les } 30 \text { min, diminution } \\
\text { plus importante du cortisol } \\
\text { dans G1 que dans G2 (-1,45 } \\
\text { vs }-0,79 \text { nmol/l). L'humeur } \\
\text { positive a augmenté de }+9,2 \\
\% \text { dans G1 alors qu'il n'y } \\
\text { avait pas de changement dans } \\
\text { G2. }\end{array}$ \\
\hline $\begin{array}{l}\text { Young et al. } \\
(2020)(47)\end{array}$ & $\begin{array}{l}\text { Zurich, } \\
\text { Suisse }\end{array}$ & $\begin{array}{l}\text { Transversal, } \\
\text { échantillon } \\
\text { aléatoire de type } \\
\text { multi-stage }^{c}, \mathrm{GC}\end{array}$ & $\begin{array}{l}\mathrm{n}=301 \text { adultes: } \\
\mathrm{G} 1: \text { jardiniers dans des } \\
\text { jardins familiaux }(\mathrm{n}=108) \\
\text { G2: jardiniers dans des } \\
\text { jardins privés }(\mathrm{n}=193)\end{array}$ & $\begin{array}{l}\text { G1: } 59 \text { ans, } \\
48 \% \mathrm{~F} \\
\text { G2: } 54 \text { ans, } \\
67 \% \mathrm{~F}\end{array}$ & $\begin{array}{l}\text { Temps moyen } \\
\text { passé dans le } \\
\text { jardin : } 17,6 \\
\mathrm{j} / \text { mois }\end{array}$ & $\begin{array}{l}\text { Santé mentale: } \\
\text { - Restauration autodéclarée } \\
(1-\text { item } Q) \\
\text { - Effets réparateurs du jardin perçus (Perceived } \\
\text { Restorativeness Scale) } \\
\text { - Stress lié au jardinage } \\
(1 \text { item) }\end{array}$ & $\begin{array}{l}\text { Les effets réparateurs du } \\
\text { jardin sont plus élevés dans } \\
\text { G1 que dans G2 }(4.72 \pm 0.47 \\
\text { vs } 4.28 \pm 0.79) \text {, ces derniers } \\
\text { étant associés à un niveau de } \\
\text { restauration plus élevé. } \\
\text { Niveau de stress plus faible } \\
\text { dans G1 que dans G2 }(2.15 \pm \\
1.23 \text { vs } 2.54 \pm 1.05) \text {. }\end{array}$ \\
\hline $\begin{array}{l}\text { Zick et al. } \\
\text { (2013)(42) }\end{array}$ & $\begin{array}{l}\text { Salt Lake } \\
\text { City, UT, } \\
\text { USA }\end{array}$ & $\begin{array}{l}\text { Evaluation post- } \\
\text { test uniquement, } \\
\text { échantillon de } \\
\text { convenance, GC } \\
\end{array}$ & $\begin{array}{l}\mathrm{n}=13133 \text { adultes: } \\
\mathrm{G} 1: \text { jardiniers dans des } \\
\text { jardins partagés }(\mathrm{n}=198) \\
\mathrm{G} 2 \text { : voisins non jardiniers }(\mathrm{n} \\
\end{array}$ & $\begin{array}{l}\text { «milieu de la } \\
40^{\text {aine jusqu'au }} \\
\text { début de } \\
50^{\text {aine }},\end{array}$ & \begin{tabular}{|l}
$>1$ an de \\
jardinage entre \\
1995 and 2010
\end{tabular} & $\begin{array}{l}\text { Anthropométrie : } \\
\text { IMC (poids et taille déclarés) }\end{array}$ & $\begin{array}{l}\text { Les femmes du G1 ont } 46 \% \\
\text { de risque en moins d'être en } \\
\text { surpoids ou obèses que leurs } \\
\text { voisines du G2. Les hommes } \\
\end{array}$ \\
\hline
\end{tabular}




$$
\text { = } 12 \text { 552) }
$$

G3: frères/sœurs non

jardinier(e)s $(\mathrm{n}=316)$

G4: époux/épouses non

jardinier(e)s $(n=67)$
$61,1 \% \mathrm{~F}$

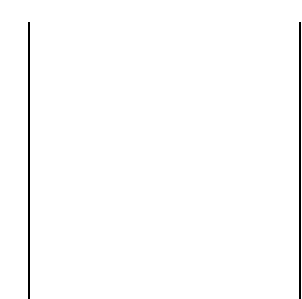

du G1 avaient $62 \%$ de risque en moins d'être en surpoids que G2 du même sexe, et 37 $\%$ de risque en moins que $\mathrm{G} 3$ du même sexe. Pas de différence d'IMC entre G1 et G4.

a Abréviations : AP : activité physique ; Q : questionnaire ; GC : groupe de comparaison

${ }^{\mathrm{b}}$ Seules les différences qui étaient statistiquement significatives sont décrites

${ }^{\mathrm{c}}$ Méthode d'échantillonnage aléatoire à plusieurs degrés permettant d'augmenter la proportion d'une catégorie peu représentée dans la population (ici les jardiniers) 
Tableau 3. Qualité méthodologique des études selon l'outil EPHPP

\begin{tabular}{|c|c|c|c|c|c|c|}
\hline & $\begin{array}{l}\text { Biais de } \\
\text { sélection }\end{array}$ & $\begin{array}{c}\text { Design de } \\
\text { l'étude }\end{array}$ & $\begin{array}{c}\text { Facteurs de } \\
\text { confusion }\end{array}$ & $\begin{array}{c}\text { Conduite en } \\
\text { aveugle }\end{array}$ & $\begin{array}{c}\text { Collecte des } \\
\text { données }^{\mathrm{a}}\end{array}$ & Taux d'attrition \\
\hline Alaimo et al. (2008)(38) & Faible & Faible & Bonne & Moyenne & Bonne & $\mathrm{n} / \mathrm{a}$ \\
\hline Alaimo et al. (2010)(34) & Moyenne & Faible & Bonne & Faible & Bonne & $\mathrm{n} / \mathrm{a}$ \\
\hline Alger et al. (2016)(39) & Faible & Faible & Faible & Faible & Moyenne & $\mathrm{n} / \mathrm{a}$ \\
\hline Booth et al. (2018)(32) & Faible & Faible & Moyenne & Faible & Moyenne & $\mathrm{n} / \mathrm{a}$ \\
\hline Comstock et al. (2010)(40) & Faible & Faible & Bonne & Moyenne & Bonne & $\mathrm{n} / \mathrm{a}$ \\
\hline Hawkins et al. (2011)(43) & Faible & Faible & Faible & Faible & Bonne & $\mathrm{n} / \mathrm{a}$ \\
\hline Litt et al. (2011)(41) & Faible & Faible & Bonne & Moyenne & Bonne & $\mathrm{n} / \mathrm{a}$ \\
\hline Litt et al. (2017)(35) & Faible & Faible & Bonne & Moyenne & Moyenne & $\mathrm{n} / \mathrm{a}$ \\
\hline Martin et al. (2017)(46) & Faible & Faible & Faible & Faible & Moyenne & $\mathrm{n} / \mathrm{a}$ \\
\hline Mourão et al. (2018)(36) & Faible & Faible & Faible & Faible & Bonne & $\mathrm{n} / \mathrm{a}$ \\
\hline Soga et al. (2017)(48) & Faible & Faible & Bonne & Faible & Bonne & $\mathrm{n} / \mathrm{a}$ \\
\hline Van den Berg et al. (2010)(33) & Faible & Faible & Bonne & Faible & Moyenne & $\mathrm{n} / \mathrm{a}$ \\
\hline Van den Berg et al. (2011)(50) & Faible & Bonne & Bonne & Faible & Bonne & Bonne \\
\hline Young et al. (2020)(47) & Faible & Faible & Bonne & Faible & Bonne & $\mathrm{n} / \mathrm{a}$ \\
\hline Zick et al. (2013)(42) & Moyenne & Moyenne & Moyenne & Moyenne & Moyenne & Bonne \\
\hline Koay et al. (2020)(49) & Faible & Faible & Bonne & Moyenne & Bonne & $\mathrm{n} / \mathrm{a}$ \\
\hline Swami et al. (2020)(44) & Moyenne & Faible & Bonne & Faible & Bonne & Bonne \\
\hline Tharrey et al. (2020)(45) & Moyenne & Moyenne & Bonne & Faible & Bonne & Bonne \\
\hline
\end{tabular}

${ }^{a}$ Les études ayant au moins la moitié des outils de mesure utilisés valides et fiables ont été considérées comme étant de bonne qualité. Les études ayant moins de la moitié des outils de mesure utilisés valides et fiables ont été considérées comme étant de qualité modérée. 